Check for updates

Cite this: RSC Adv., 2018, 8, 33103

Received 6th July 2018

Accepted 14th September 2018

DOI: $10.1039 / c 8 r a 05763 j$

rsc.li/rsc-advances

\title{
A hybrid micromixer with planar mixing units $\dagger$
}

\author{
Sajad Razavi Bazaz, ${ }^{\text {ab }}$ Ali Abouei Mehrizi, ${ }^{\text {bb }}$ Sadegh Ghorbani, ${ }^{c}$ Steven Vasilescu, ${ }^{d}$ \\ Mohsen Asadnia (D) and Majid Ebrahimi Warkiani (D) *af
}

The application of microfluidic systems in chemical and biological assays has progressed dramatically in recent years. One of the fundamental operations that microfluidic devices must achieve is a high mixing index. Of particular importance is the role of planar mixing units with repetitive obstacles (MURO) in the formation of micromixers. To date, a myriad of planar passive micromixers has been proposed. However, a strategy for the combination of these units to find an efficient planar mixer has not been investigated. As such, five different MURO have been selected to form a "hybrid micromixer," and their combination was evaluated via numerical and experimental methods. These mixing units include ellipse-like, Tesla, nozzle and pillar, teardrop, and obstruction in a curved mixing unit. Since these units have distinctive dimensions, dynamic and geometric similarities were used to scale and connect them. Afterwards, six slots were designated to house each mixing unit. Since the evaluation of all possible unit configurations is not feasible, the design of experiment method is applied to reduce the total number of experiments from 15625 to 25. Following this procedure, the "hybrid" micromixer proposed here, comprising Tesla, nozzle and pillar, and obstruction units, shows improved performance for a wide range of Re (i.e., mixing index of $>90 \%$ for $\operatorname{Re} 0.001-0.1,22-45)$ over existing designs. The use of velocity profiles, concentration diagrams, vorticity and circulation plots assist in the analysis of each unit. Comparison of the proposed "hybrid" micromixer with other obstacle-based planar micromixers demonstrates improved performance, indicating the combination of planar mixing units is a useful strategy for building high-performance micromixers.

\section{Introduction}

There have been many studies recently investigating the implementation of microfluidic systems in biological and chemical assays. Microfluidic systems offer several advantages over lab scale operations stemming from elevated heat and mass transfer; a result of high surface area to volume ratios. Higher levels of sensitivity, fast and straightforward fabrication as well as the practicability of in situ operation make them ideal. ${ }^{1}$ The future of biological research could greatly benefit from microfluidic devices, among which micromixers-exhibiting precise and controllable features-have manifold practical

${ }^{a}$ School of Biomedical Engineering, University of Technology Sydney, New South Wales 2007, Australia.E-mail: majid.Warkiani@uts.edu.au

${ }^{b}$ Biomedical Engineering Division, Department of Life Science Engineering, Faculty of New Sciences and Technologies, University of Tehran, Tehran, Iran. E-mail: abouei@ut.ac.ir

'Department of Anatomical Sciences, Faculty of Medicine, Tarbiat Modares University, Tehran, Iran

${ }^{d}$ Faculty of Science, University of Technology Sydney, New South Wales 2007, Australia ${ }^{e}$ Department of Engineering, Faculty of Science and Engineering, Macquarie University, Sydney, NSW 2109, Australia

${ }^{f}$ Institute of Molecular Medicine, Sechenov University, Moscow 119991, Russia

$\dagger$ Electronic supplementary information (ESI) available. See DOI: $10.1039 / \mathrm{c} 8 \mathrm{ra05763j}$ applications. $^{2}$ Micromixer applications are not limited to chemical synthesis; they can also contribute to DNA purification, synthesis of nucleic acids, cell lysis, drug delivery, biological analysis, and polymerase chain reaction (PCR). ${ }^{3,4}$ For example, Kim et al. reported the use of micromixers to enhance the mixing of glucose and glucose oxidase in chemical synthesis. Their results indicated a mixing index of 81 to $91 \%$ within a range of 0.08 to 16 Reynolds numbers (Re). ${ }^{5}$ In another study, Kim et al. makes use of rapid micromixers to conduct an investigation in organic synthesis; the reported mixing index and resulting product yield were $80 \%$ and $91 \%$, respectively. ${ }^{6}$ Micromixers are also critical in microreactors. Ko and colleagues utilized a micromixer with different nozzle and pillar units for DNA ligation where the maximum mixing efficiency was reported to be $87.7 \%$ in Re of $66.5 .^{7}$ Tobias et al. used an active micromixer to investigate the relation between metastasizing of tumors and the formation of thrombosis. ${ }^{8}$ Nason et al. used a zigzag micromixer for biological analysis, specifically drug screening, where the mixing index was reported as $79.4 \% .^{9}$ Micromixers continue to be applied to a diverse range of applications from biological analysis to chemical synthesis.

Micromixers typically fall in the two categories of active and passive. ${ }^{\mathbf{1 0}}$ Active micromixers rely on external forces such as thermal, acoustic, magnetic, and electro-kinetic to improve the mixing efficiency while passive micromixers do not need an 
external power source. ${ }^{11}$ Although active micromixers have high mixing efficiency, their power requirements and added complexity make them a less viable option. In contrast, passive micromixers rely on chaotic advection by way of their geometry to stretch, fold, split, or break the flow of fluids. Furthermore, they also rely on molecular diffusion by lamination of the flow and an increase the surface area between fluid layers. These effects afford more versatility in this regard. ${ }^{\mathbf{1 2}}$ They can be easily integrated with other microfluidic components such as micropumps and microvalves in order to make a lab-on-a-chip device, "a device which integrates several distinct experimental func-

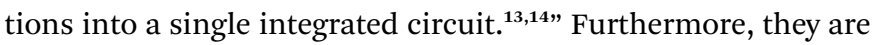
easily fabricated and do not require an external power/force to manipulate the fluid. Passive micromixers do not always require low Re or a fixed flow rate for operation. ${ }^{15}$ Hence, an adaptable micromixer with an appropriate mixing index for a range of different Re should be proposed.

Based on the channel geometry, passive micromixers are divided into two groups. The first group consists of devices with either simple or complicated geometries with no repetitive design patterns. In this group, the geometry is manipulated to create either Dean forces or chaotic advection to increase molecular diffusion and in turn the mixing efficiency. The second group has two or more mixing units that repeat along the length of the microchannel. Generally, passive micromixers with mixing units can be categorized into three separate classes: planar mixing units with repetitive obstacles (MURO), planar mixing units with repetitive units (MURU), and mixing units with repetitive 3D units (MUR3DU). Generally, micromixers whose height changes with length are defined as 3D micromixers. ${ }^{16}$ In these types of micromixers, the $Z$-direction plays a significant role in mixing efficiency as it impacts chaotic advection and Dean forces. On the other hand, micromixers which have the same height are referred to as planar micromixers. The salient feature of mixing units with 3D units is their high mixing index. However, the complicated fabrication process of these units as well as the time-consuming process for integration with other components (in a lab-on-a-chip device) are considerable issues. In MURU, stream flow varies with the geometry of the channel while no additional obstacles are being used. These kinds of mixing units chiefly use split and recombine (SAR) techniques in which the flow patterns separate into sub-channels, and later re-combine. Since the geometry manipulation is the only variable of these mixing units, it is often difficult to reach the desired mixing efficiency. Furthermore, in some cases, an increase in geometric complexity may complicate the fabrication processes. Micromixers with MURO have a specific patterned obstacle which is repeated along the length of the microchannel. Based on their shape, obstacles can break the flow pattern of the stream and create additional vortices and enhance chaotic advection. Therefore, the mixing index of MURO is significantly higher than MURU, making them the favorable choice for planar mixing units.

A review of relevant publications reveals that numerous mixing units have been proposed. Elliptical units, ${ }^{17}$ Tesla units, ${ }^{18}$ E-shaped mixing units, ${ }^{19}$ F-shaped mixing units, ${ }^{20}$ and $\mathrm{J}$ shaped mixing units ${ }^{21}$ are just a few mixing units with obstacles that have been named. In some cases, a unit is proposed, and then, several groups work to improve its efficiency regarding their mixing units, pressure drop, mixing time, and so forth. For example, rhombic mixing units, which fall into the MURU category, were initially proposed by C. K. Chung et al. ${ }^{22}$ inspired from a zigzag microchannel. This lead to the publication of more than six papers working with this type of mixing unit. Among those papers, one of them added obstacles in the microchannel, ${ }^{23}$ while another one appended an extra subbranch. ${ }^{24}$ Shakhawat Hossain et al. utilized three-split rhombic sub-channels to increase the mixing efficiency. ${ }^{25}$ Although numerous mixing units are offered, a combination of these units together is not investigated, to date. Since there are myriad ways to unite certain nominated MURO together, a solution has to be implemented to address this issue. As a result, the design of experiment (DOE) method is employed to organize the combination of mixing units and reduce the total number of experiments (please refer to $\mathrm{ESI} \dagger$ for a detailed history of DOE).

The primary aim of this study is to investigate the combination of different MURO to form a "hybrid" micromixer for a wide range of applications. At first, nominated planar mixing units were delineated. Then, the appropriate method of DOE was employed to investigate the combination of these units. In order to connect these units together efficiently and correctly, dynamic and geometric similarities were utilized. Since obtaining experimental results for a large number of experiments is time consuming, labour intensive, and costly, a validation process has been performed in order to be confident in the CFD codes. A number of combined micromixers were fabricated, tested, and investigated during the entire process. Ensuing analysis using the $\mathrm{S} / \mathrm{N}$ ratio diagrams allowed us to determine the optimum results of the MURO. After selecting the optimized micromixer with MURO, as the "hybrid" micromixer, the impact of each mixing unit on the total performance of the micromixer was carefully investigated. This investigation yielded a variety of results that have been depicted. Following these processes, the function of the "hybrid" micromixer over a wide range of applications was carefully evaluated.

\section{Modeling assumptions and fabrication process}

Firstly, the theoretical background behind the micromixer designs, including the governing equations, will be presented. This is followed by the description of DOE and the selected geometries of the MURO, as well as the fabrication process used in this study.

\subsection{Theoretical aspect of the "hybrid" micromixer}

2.1.1 Dynamic and geometric similarity. Since the different mixing units do not have identical width $(W)$, height $(H)$, and hydraulic diameter $\left(D_{\mathrm{h}}\right)$, selected mixing units need to be scaled in order to classify them. In order to attain perfect similarity between the scaled micromixer (SM) and the chosen micromixer (CM), both dynamic and geometric similarities are 
required. Geometric similarity happens when uniform scaling occurs at the centroid region. It means that the relation between each part of the model has to be kept equal with the scaled one. Eqn $(1 \mathrm{a}-\mathrm{c})$ show how this similarity can be evaluated.

$$
\begin{aligned}
& \left(\frac{D_{\mathrm{h}}}{L}\right)_{\mathrm{SM}}=\left(\frac{D_{\mathrm{h}}}{L}\right)_{\mathrm{CM}} \\
& \left(\frac{W}{L}\right)_{\mathrm{SM}}=\left(\frac{W}{L}\right)_{\mathrm{CM}} \\
& \left(\frac{H}{L}\right)_{\mathrm{SM}}=\left(\frac{H}{L}\right)_{\mathrm{CM}}
\end{aligned}
$$

Due to the effects of mass advection and mass diffusion during mixing, the velocity and concentration are two vital parameters in the mixing process. As a result, two dimensionless numbers (either of which is responsible for one vital parameter) are applied to evaluate the dynamic similarity. The first one is Re which indicates the ratio of inertia to viscous forces and is defined by eqn (2).

$$
\operatorname{Re}=\frac{\rho V D_{\mathrm{h}}}{\mu}
$$

where, $\rho$ is the density, $\mu$ is the dynamic viscosity, and $V$ is the velocity. The main function of Re is to assess the fluid characteristics in the channels. The second dimensionless number is the Péclet number $(\mathrm{Pe})$ which represents the ratio of convective to diffusive mass transfer; its function is to evaluate the mixing process and is indicated by eqn (3).

$$
\mathrm{Pe}=\frac{V D_{\mathrm{h}}}{D}
$$

where, $D$ is the diffusion coefficient.

Re in the SM and CM must be similar in order to confirm identical fluid characteristics. By using fluids in the SM and CM with the exact same physical properties, eqn (4) will be obtained.

$$
(\mathrm{Re})_{\mathrm{SM}}=(\mathrm{Re})_{\mathrm{CM}} \rightarrow\left(V D_{\mathrm{h}}\right)_{\mathrm{SM}}=\left(V D_{\mathrm{h}}\right)_{\mathrm{CM}}
$$

Pe will be achieved if the diffusion coefficient is divided in both sides. Thus, if Re is the same in both the SM and CM, Pe will be maintained, and the dynamic similarity can be confirmed.

2.1.2 Governing equations and boundary conditions. In this study, simulations were carried out using COMSOL Multiphysics 5.2a, a commercial software based on the finite element method. All simulations were implemented by three separate computers (the first of which was controlled remotely) containing an Intel Xeon E312 (Sandy Bridge) processor and installed memory of $31.0 \mathrm{~GB}$ on a 64 -bit operating system, a desktop computer with an Intel Core i7-3610QM $2.30 \mathrm{GHz}$ processor integrated with $8.00 \mathrm{~GB}$ RAM on a 64-bit operating system, and a desktop computer with an Intel Core i5-5200U $2.20 \mathrm{GHz}$ processor with installed memory of $6.00 \mathrm{~GB}$ on a 64bit operating system and a x64-based processor. The micromixer models were simulated by solving eqn (5)-(7) numerically which are the continuity, Navier-Stokes, and convection-diffusion equations, respectively.

$$
\begin{gathered}
\nabla \cdot \boldsymbol{V}=0 \\
\frac{\partial \boldsymbol{V}}{\partial t}+\rho(\boldsymbol{V} \cdot \nabla) \boldsymbol{V}=-\nabla P+\mu \nabla^{2} \boldsymbol{V} \\
\frac{\partial c}{\partial t}+(\boldsymbol{V} \cdot \nabla) c=\frac{1}{\operatorname{ReSc}} \nabla^{2} c
\end{gathered}
$$

where, $\boldsymbol{V}$ is the velocity vector, $P$ is the fluid pressure, $c$ is the concentration of species, and Sc is Schmidt number which indicates the ratio of momentum and mass diffusivity and can be obtained by eqn (8).

$$
\mathrm{Sc}=\frac{\nu}{D}=\frac{\mu}{\rho D}
$$

where, $\nu$ is the kinematic viscosity. The flow was considered as a steady-state, incompressible, and Newtonian flow with a density of $988 \mathrm{~kg} \mathrm{~m}^{-3}$ and dynamic viscosity of $0.00089 \mathrm{~Pa}$. Uniform velocity had been assigned to both inlets, and the zero static pressure had been set to the outlet. No-slip boundary condition had been applied to the walls. To evaluate this, Knudsen number $(\mathrm{Kn})$ was used. $\mathrm{Kn}$ is a dimensionless number used to distinguish the type of slip boundary condition and is defined by eqn (9). ${ }^{26}$

$$
\mathrm{Kn}=\frac{\lambda}{L_{\mathrm{c}}}
$$

where, $\lambda$ is the mean free path length and $L_{\mathrm{c}}$ is the characteristic length of microchannel. No-slip boundary condition will be applied if the Knudsen number is lower than $10^{-3}$. Since the flow characteristics of this study were approximately similar to that of water, $\lambda$ of water $(0.25 \mathrm{~nm})$ was used to calculate $\mathrm{Kn}$ number, which was approximately $1.7 \times 10^{-6}$. As a result, the continuum theory and no-slip boundary condition for the walls were deemed satisfactory. The diffusivity between the fluids tested was assumed to be $2 \times 10^{-9} \mathrm{~m}^{2} \mathrm{~s}^{-1}$. The molar concentration of the two species was set as 0 and 1 , and uniform mixing was achieved when the molar intensity of two species reached the value of 0.5 . In order to ascertain the mixing index as a standard criterion for evaluation of the mixing process, eqn (10) was used.

$$
\mathrm{MI}=1-\sqrt{\frac{1}{n} \sum_{i=1}^{n}\left(\frac{k_{i}-\bar{k}}{\bar{k}}\right)^{2}}
$$

In eqn (10), MI is the mixing index, $n$ is the total numbers of sample points, $k_{\mathrm{i}}$ is the mole fraction over the outlet crosssection, and $\bar{k}$ is the average mole fraction. The value of MI varies between 0 and 1 where $\mathrm{MI}=0$ ( $0 \%$ mixing) indicates no mixing, while $\mathrm{MI}=1$ represents complete mixing (100\% mixing). The transverse diffusion time can be calculated by eqn (11).

$$
t=\frac{L_{\mathrm{c}}^{2}}{D}
$$


where, $L_{\mathrm{c}}$ for the non-circular pipe is the hydraulic diameter. The characteristic mixing length for the micromixer to reach complete mixing can be represented by eqn (12).

$$
L=V t=V \frac{L_{\mathrm{c}}^{2}}{D}=\operatorname{Pe} L_{\mathrm{c}}
$$

Since Pe is directly related to $V$, by increasing Pe, $V$ will be increased, and as a result, so will Re. Eqn (12) illustrates that a higher Pe is related to mixing as a result of diffusion for a limited range of $L$. This condition is true if the value of Re is less than the critical point in which the turbulent flow occurs or if it is unable to generate additional vortices and chaotic advection. Higher values for Re contribute to a higher Pe and as a result, the MI is decreased. The critical value of Re is contingent on the geometry of the mixer and the velocity of the flow. After the critical point, when streams reach planted obstacles, the laminar flow breaks up thereby inducing the formation of a secondary flow field, detaching the boundary layer, and generating the vortices. The vortices generated a curling action in which the stream broke up into layers and the diffusion distance between the molecules of liquids decreased. During this process, mass advection was dominant and the passive method for mixing proved advantageous for chaotic advection.

\subsection{Design of experiment and the geometry of selected mixing units with obstacles}

For the aim of forming a "hybrid" micromixer, five MURO were selected, each of which had a unique feature. It is of significant importance to mention that these units have to approximately carry the same volume of samples; otherwise, outliers in the result of DOE as a statistical approach occur. In addition, if a unit from a famous family had been selected, meticulous attention had to be paid to whether the latest generation of this unit had been selected or not. Furthermore, inherent features of units must remain intact. For instance, in some cases, when the flow passed the first mixing unit, the flow changed 90 degrees counterclockwise or clockwise, or the direction of the mixing unit changed from upward to downward, or vice versa. In all cases, this feature for the "hybrid" micromixer had to be maintained. Following an investigation into MURO, five units, each of which is illustrated in Fig. 1, were selected. The first selected MURO was an ellipse-like mixing unit. ${ }^{17}$ In the original study, Nhut Tran-Minh used ten mixing units which were connected together with a link to build a micromixer. The crosssection of the outlet of each unit was smaller than that of the inlet of each unit, and this led to an increase in the outlet velocity. Therefore, at the outlet of each unit, two flows were recombined with high velocity, so that the mixing index increased. The second MURO chosen was circular in shape with obstructions in a curved microchannel. The microchannel connected seven units together in which the first one was orientated upward, the second one was downward, and others followed the same pattern. ${ }^{27}$ As we aimed to have a combined micromixer with minimal length, the selection of one unit informed the selection of the following unit. When considering mixing units, two categories exist. The first is non-directional, whereas the second may be orientated upwards or downwards about the center axis. If a directional unit with an upward pattern is selected, the following directional unit must be orientated downwards, and vice versa. Therefore, in order to minimize the length of the micromixer, directional units were not immediately repeated.

The third unit was Tesla mixing unit. Tesla structure had formerly been utilized in in-plane microvalves and micropumps. ${ }^{28,29}$ In 2003, Chien-Chong Hong et al. proposed a micromixer in which a Tesla structure was used..$^{18}$ Then, Ali Asgar S. Bhagat used this structure to enhance particle dispersion in his designed micromixer. ${ }^{30}$ Shakhwat Hossain showed by a parametric study that the pressure drop and the mixing index in the Tesla structure strictly depended on geometrical parameters. He opted for a specific method where he optimized the parameters of Tesla structure, ${ }^{31}$ obtaining the scaled Tesla structure illustrated in Fig. 1. The fourth mixing unit was a mixture of nozzle and pillar mixing units which was proposed by Yong-Jun Ko et al. ${ }^{7}$ The device had been mainly applied for DNA ligation. In the mentioned study, three micromixers with separate mixing units were tested and the appointed one showed a meaningful mixing index in high Re numbers, which was employed for this study. Similar to the second selected unit, this unit arrangement was also upward-downward. Therefore, its direction changed when flow passed through it. The last selected unit was a unit with teardrop obstruction elements. Kristina J. Cook et al. in her study employed uneven interdigital inlets to investigate her proposed micromixer for the Re ranging between 1 and $100 .{ }^{32}$ A series of obstacles in the shape of a teardrop were used to increase the mixing efficiency. Fig. 1 demonstrates the nominated mixing units after scaling from their centroid with two extra views and an isometric 3D view.

Although increasing the number of mixing units leads to an increase in the mixing index, the total length of micromixer is important as it has to be integrated with other parts of a lab-ona-chip device. Six design slots were selected for potential mixing units and all these units were linked together with a connecting channel. Since we scaled units about their centroid to successfully assemble them, the height of units differed from each other. Assembling units with different heights could cause such unpredictable and uncontrollable effects as accelerated or decelerated flows to create additional vortices and undesirable pressure drops. These devices referred to as planar micromixers, owing to the fact that the $Z$ direction was the same in each unit, experienced no transverse effect. The $Z$ direction had the least effect on the total function of each unit. Thus, a specific height of $100 \mu \mathrm{m}$ prevalent in planar MURO $^{7,12,27,33}$ was proposed for the height of all units and "hybrid" micromixer to obviate all the mentioned detrimental effects. The schematic design of the "hybrid" micromixers with MURO with all possible number of experiments is elucidated in Fig. 2a.

Fig. 2a shows that so as to find the best combination of MURO, six factors, all of which have 5 levels, exist. Therefore, a total number 15625 experiments and simulations had to be carried out which was not feasible in the real world. Since our data is categorical, Taguchi method has been employed as 

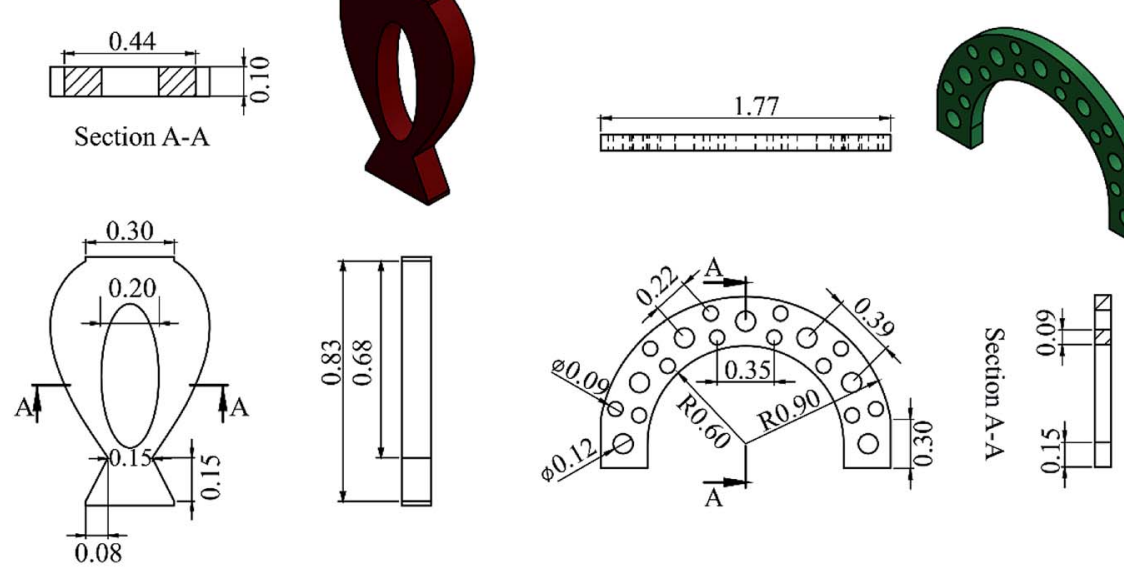

Ellipse-like Mixing Unit

\section{Obstructions in a Curved Mixing Unit}

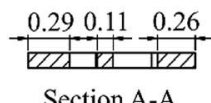

Section A-A
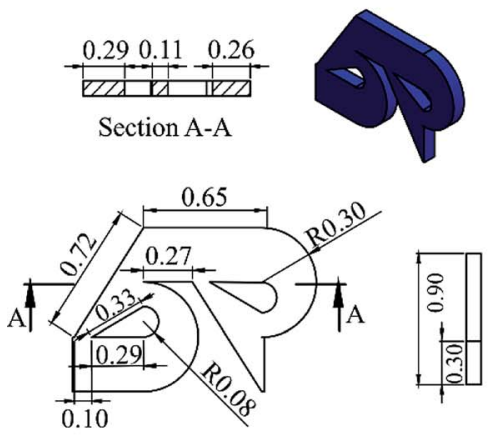

Tesla Mixing Unit

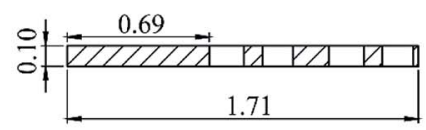

Section A-A
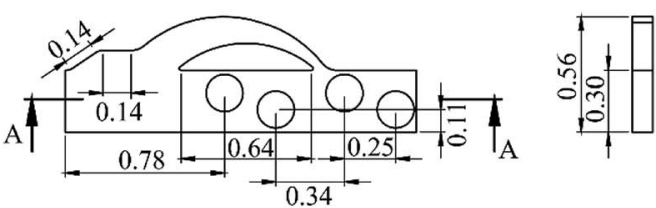

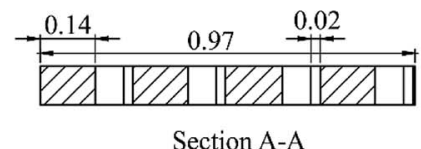

Section A-A

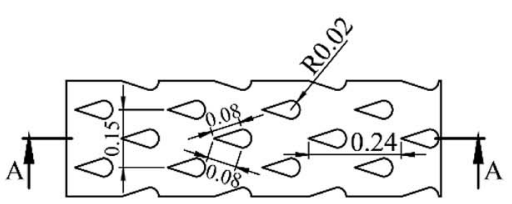

\section{Teardrop Mixing Unit}
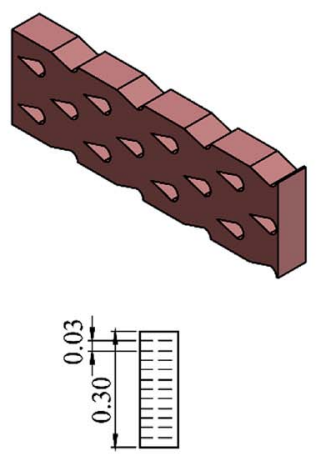

Fig. 1 Illustration of scaled nominated MURO which are ellipse-like, Tesla, nozzle \& pillar, and teardrop mixing unit as well as the obstruction in a curved mixing unit. The MURO elucidated are the components chosen to form the hybrid micromixer, the best combination of which constitutes the final design. For further clarification, two additional views of each MURO as well as the isometric view are displayed. Each unit is dimensioned in front, top and side views. All dimensions are in $\mathrm{mm}$.

a DOE. As the order of levels in Taguchi method was not important, the arrangement of factors and levels are similar to Fig. 2a. For the six factors and five levels, Taguchi offered the orthogonal array of L25 ( $5^{6}$ which 5 denotes number of factors and 6 represents number of levels for each factor) which reduced the total number of experiments from 15625 to 25 with 


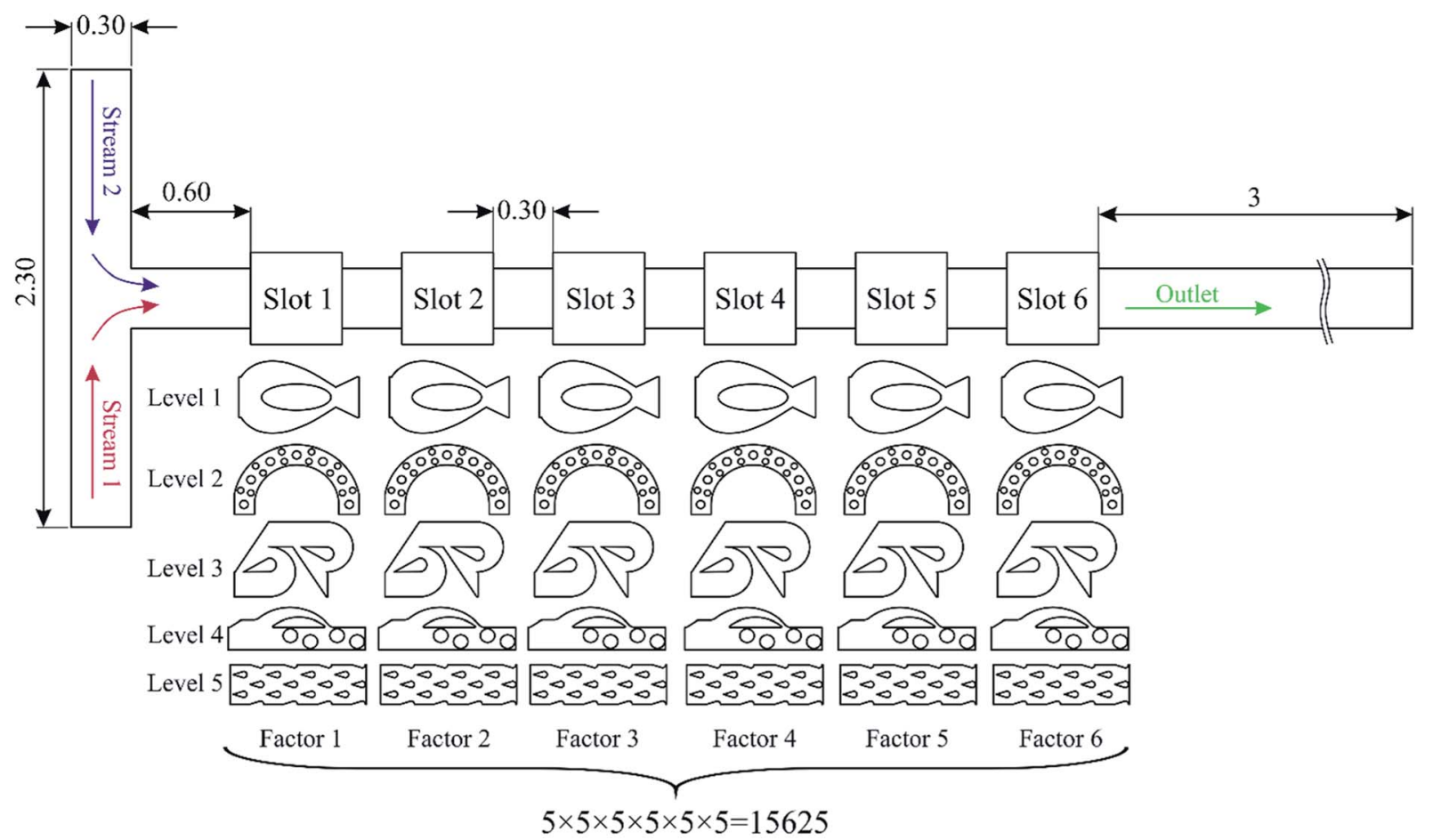

a)
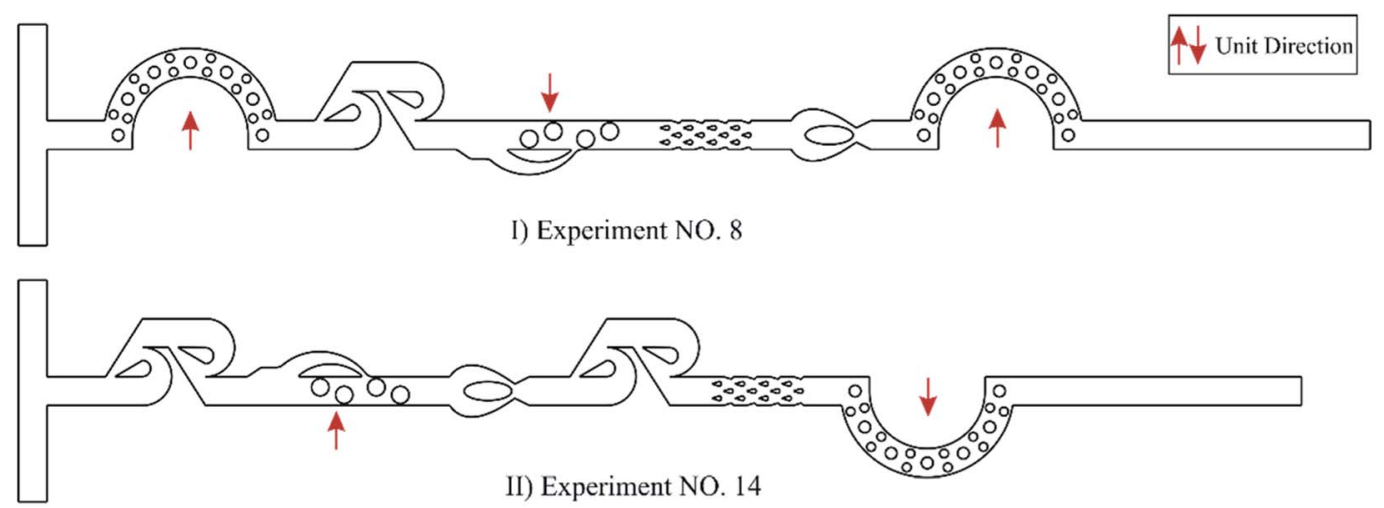

b)

Fig. 2 (a) All possible combinations of nominated MURO to be placed in each unit are displayed here. In these combinations, there are six factors in which all of them have five similar levels. Each MURO is connected by $0.3 \mathrm{~mm}$ links. (b) Two sample configurations of micromixers with MURO based on Taguchi table are supplied as examples. Red arrows show the unit direction, and all unit directions are assumed to be upward at first. All dimensions are in $\mathrm{mm}$.

its specific combination. The detailed table of Taguchi is indicated in Table 1 where the numbers indicate the level (mixer type) based on Fig. 2. (Please refer to ESI $\dagger$ for a comprehensive description of Taguchi method)

Drawing of all mixing units as well as assembly was undertaken in SolidWorks 2016, commercial CAD/CAE software. Special care was taken when assembling these units so that units 2 and 4 have the sequence of upward-downward, and their direction changes when flow passed through them. The first direction of both of them was upward. To better illustrate, two models among the 25 models are shown in Fig. $2 \mathrm{~b}$, and the others follow this protocol.

\subsection{Fabrication process and mixing experiments}

Observing the accuracy of the numerical results led us to perform experiments on specific devices. The fabrication of the micromixers was done in accordance with the identified geometric dimensions with standard photolithography technique. Afterwards, the developed PDMS and the glass were bonded together by oxygen plasma treatment. The microchips were monitored through a CCD camera mounted on an optical microscope (Olympus, Japan). Two samples were simultaneously introduced from inlets at the same Re using a syringe pump (Harvard Co., USA). Then, the images and the related video files were separately captured along the length of the 
Table 1 The proposed table of Taguchi for six factors and five levels

Slots

\begin{tabular}{lllllll} 
Experiments & Slot 1 & Slot 2 & Slot 3 & Slot 4 & Slot 5 & Slot 6 \\
\hline
\end{tabular}

\begin{tabular}{|c|c|c|c|c|c|c|}
\hline 1 & 1 & 1 & 1 & 1 & 1 & 1 \\
\hline 2 & 1 & 2 & 2 & 2 & 2 & 2 \\
\hline 3 & 1 & 3 & 3 & 3 & 3 & 3 \\
\hline 4 & 1 & 4 & 4 & 4 & 4 & 4 \\
\hline 5 & 1 & 5 & 5 & 5 & 5 & 5 \\
\hline 6 & 2 & 1 & 2 & 3 & 4 & 5 \\
\hline 7 & 2 & 2 & 3 & 4 & 5 & 1 \\
\hline 8 & 2 & 3 & 4 & 5 & 1 & 2 \\
\hline 9 & 2 & 4 & 5 & 1 & 2 & 3 \\
\hline 10 & 2 & 5 & 1 & 2 & 3 & 4 \\
\hline 11 & 3 & 1 & 3 & 5 & 2 & 4 \\
\hline 12 & 3 & 2 & 4 & 1 & 3 & 5 \\
\hline 13 & 3 & 3 & 5 & 2 & 4 & 1 \\
\hline 14 & 3 & 4 & 1 & 3 & 5 & 2 \\
\hline 15 & 3 & 5 & 2 & 4 & 1 & 3 \\
\hline 16 & 4 & 1 & 4 & 2 & 5 & 3 \\
\hline 17 & 4 & 2 & 5 & 3 & 1 & 4 \\
\hline 18 & 4 & 3 & 1 & 4 & 2 & 5 \\
\hline 19 & 4 & 4 & 2 & 5 & 3 & 1 \\
\hline 20 & 4 & 5 & 3 & 1 & 4 & 2 \\
\hline 21 & 5 & 1 & 5 & 4 & 3 & 2 \\
\hline 22 & 5 & 2 & 1 & 5 & 4 & 3 \\
\hline 23 & 5 & 3 & 2 & 1 & 5 & 4 \\
\hline 24 & 5 & 4 & 3 & 2 & 1 & 5 \\
\hline 25 & 5 & 5 & 4 & 3 & 2 & 1 \\
\hline
\end{tabular}

\section{Results and discussion}

\subsection{Analysis of Taguchi table}

Fig. 3 shows a multi-panel graph depicting 25 experimental results which were proposed by Taguchi. Since most lab-on-achip devices work with low Re, the optimization process was performed for a Re of 5, and later extended to a wide range of Re. (Please refer to the ESI $\uparrow$ for the grid study of the numerical results). Fig. 3(a-f) represent the mixing index of each experiment versus each unit in the combined micromixer. It also shows the specific number for each experiment in its legend in accordance with the Taguchi table. As can be concluded from Fig. 3, the total number of experiments were normally distributed among all factors and levels. Indeed, each level in a unit contributed to 5 experiments, and this number was the same for all units and their levels. It is seen in Fig. 3a that experiments 2, 3 , and 4 , as shown in dark blue color, have the best mixing index in unit 1 and level 1 . The combined micromixer of 2,3 , and 4 in the DOE table show that level 1 in this unit is not an effective unit, but the $2^{\text {nd }}, 3^{\text {rd }}$, and $4^{\text {th }}$ level are dominant. Therefore, for unit 1, levels 2,3 , and 4 are suitable candidates. A trenchant analysis of these six figures reveals that the mixing index is

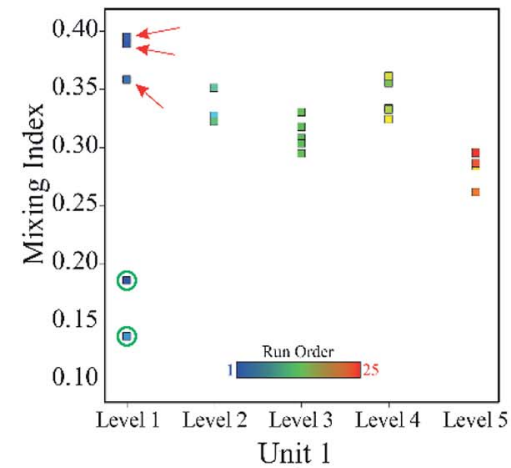

a)

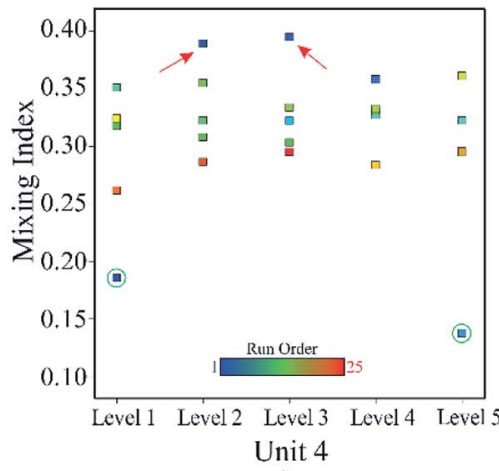

d)

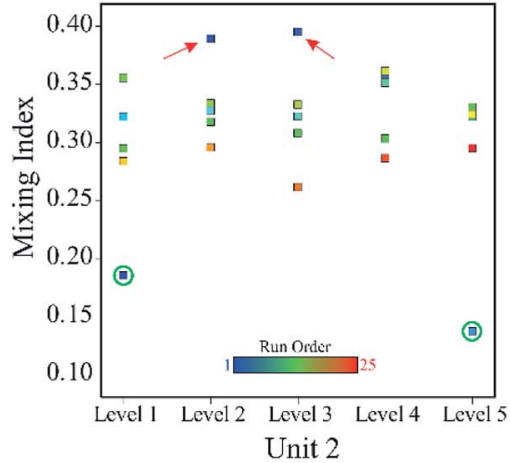

b)

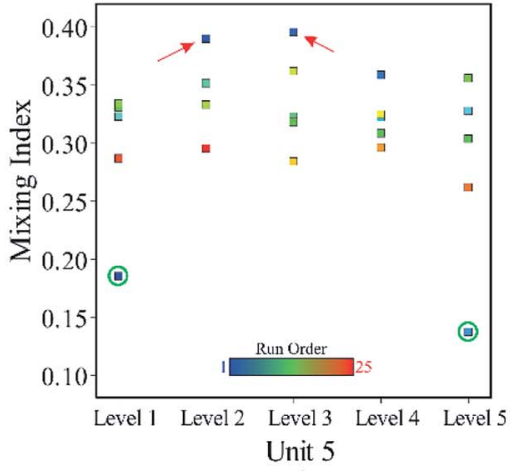

e)

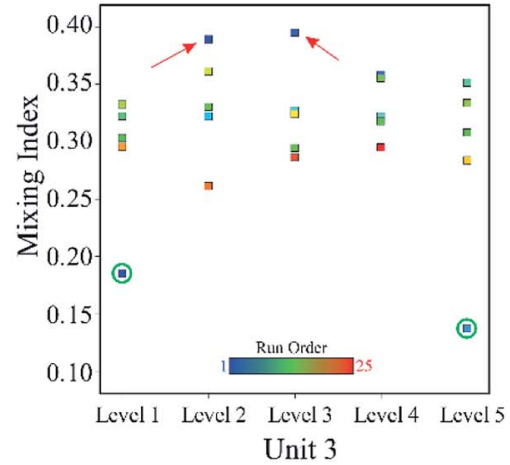

c)

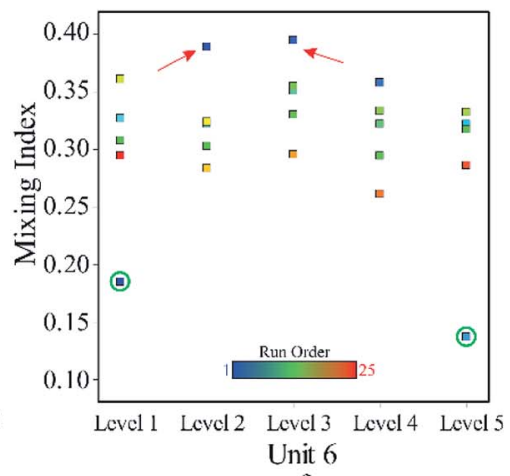

f)

Fig. $3(a-f)$ The mixing index of each simulation versus the levels of each mixing unit are compared in these six figures. To better illustrate the role of each MURO across every unit, each figure is representative of the mixing index for all possible levels of each unit. Green circles show the lowest mixing indexes while red arrows show the highest. 
smallest in the experiments where unit 1 and unit 5 play the major role as marked by a green circle. Thus, it can be approximated that the $1^{\text {st }}$ and $5^{\text {th }}$ levels of all factors can be neglected for the final selection. (Please refer to the ESI and Fig. $\mathrm{S} 2 \uparrow$ for detailed results of simulation no. 7 in the DOE table)

\subsection{Experimental results}

To justify the credibility of the numerical simulations, experimental results for certain simulations were carried out. Five different micromixers from Table 1 were randomly chosen; their simulation numbers were $7,12,16,21$, and 25 . To enhance the accuracy of experimental results, exact dimensions of the microchannel were extracted using a profilometer (Bruker, USA). Fig. 4 illustrates the images obtained from the profilometer for simulation numbers 7 and 21. (Please refer to ESI and Fig. $\mathrm{S} 3 \dagger$ for profilometer of experimental numbers 12, 16, 25 , and the "hybrid" micromixer.)

By using the parameters analyzed from the profilometer, exact dimensions of the microchip were identified, followed by the calculation of the hydraulic diameter. Therefore, the calculation of the input flow rate of the syringe pump was made as accurate as possible. Initially, in numerical simulations, original sample concentrations are displayed by solid color bands. These color bands gradually integrate as they interact with mixing units. In such cases, when the obstacles impede the path of the fluids, different layers of streams represented by different colors are discernible. It is of significant importance that the numerical simulations reflect the experimental test results. In other words, the simulations are able to predict the fluid behavior so they may be a reliable reference for future scrutiny. Accordingly, Fig. 5 shows a comparison between the experimental results and the numerical simulations in different cross-sectional areas for design numbers of 12, 16 and 25 . (Please refer to the ESI and Fig. S5 $\uparrow$ for other comparisons)

In Fig. 5, contour plots of concentration distribution extracted from numerical simulations are depicted. Then, the experimental results are illustrated in each section to compare them with the numerical ones. This analogy establishes a quantitative framework for detecting and analyzing these two kinds of results. Prior to this, it was difficult to make predictions about the reliability of the numerical results. Since Re is set at 5 , the flow regime is diffusive, and there is no trace of turbulence. In design number 12, when the streams reach the first mixing unit, they pass either side of the obstacle, splitting the fluid before recombining. As a result, the intensity of the light decreases as shown in Fig. 5. To better evaluate the CFD code, a magnified version of the experimental and numerical result is displayed. Upon comparison, a reliable correlation between simulation results and experimental data was observed; simulation models were able to accurately predict the flow behavior for experimental tests. Comparing the design numbers of 16 and 25 reveals that the flow prediction from the numerical results of design number 12 is not accidental, the proof of which can clearly be identified in Fig. 5. Taken together, the

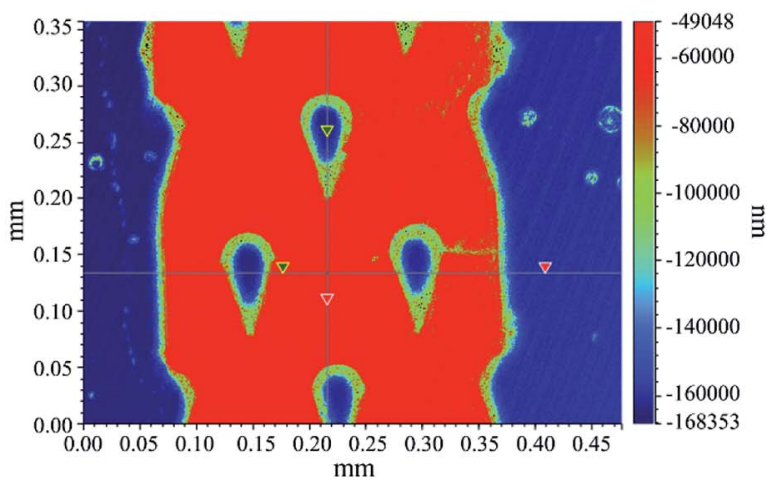

a)
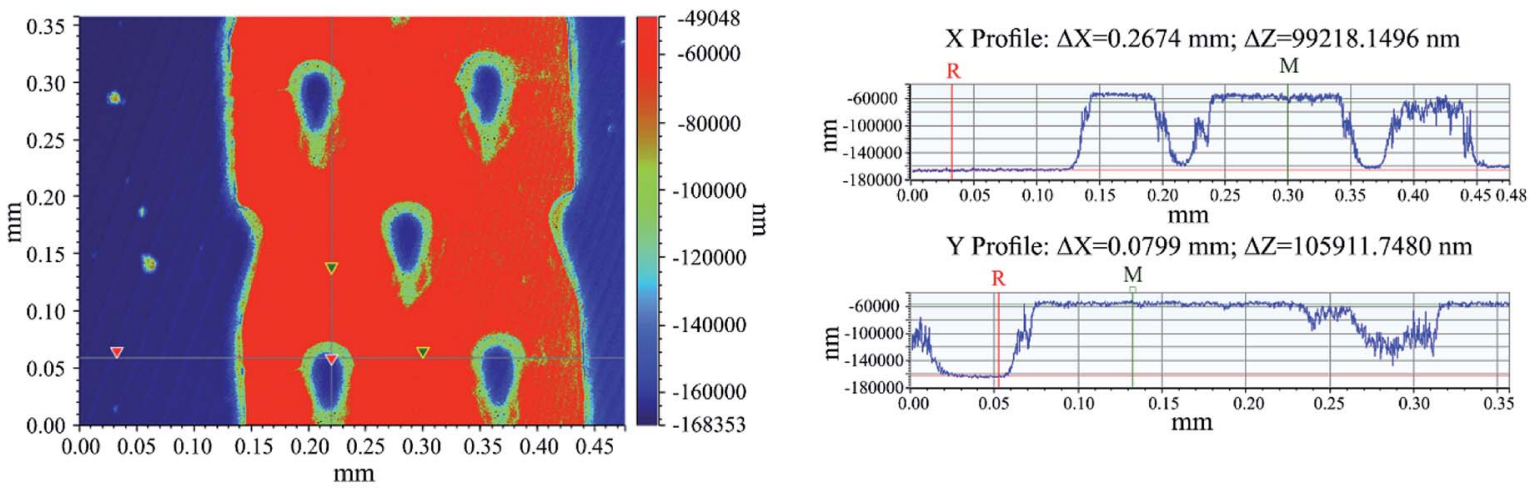

b)

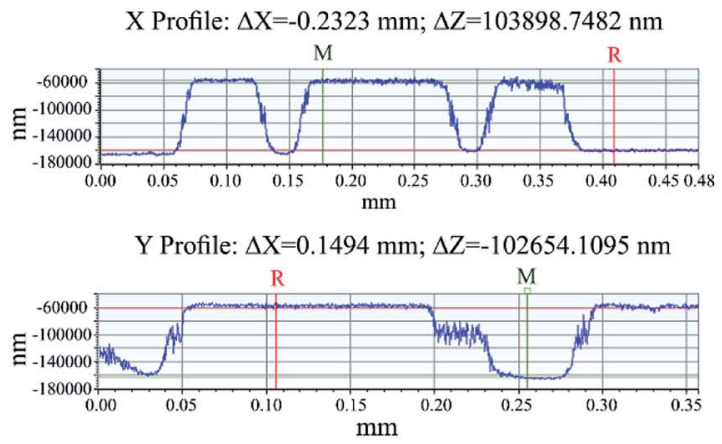

$$
\text { b) }
$$

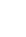

Fig. 4 Image extracted via profilometer to measuring the exact dimensions of the (a) design number 7 (b) design number 21. 


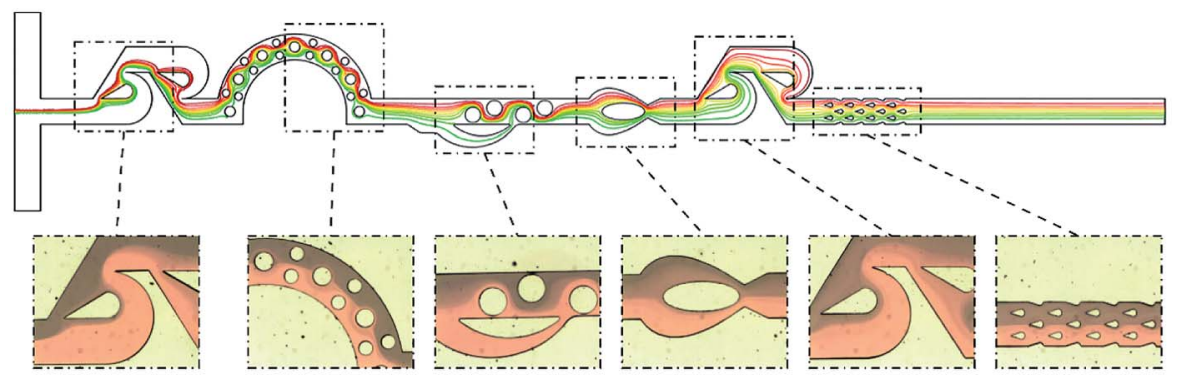

a)

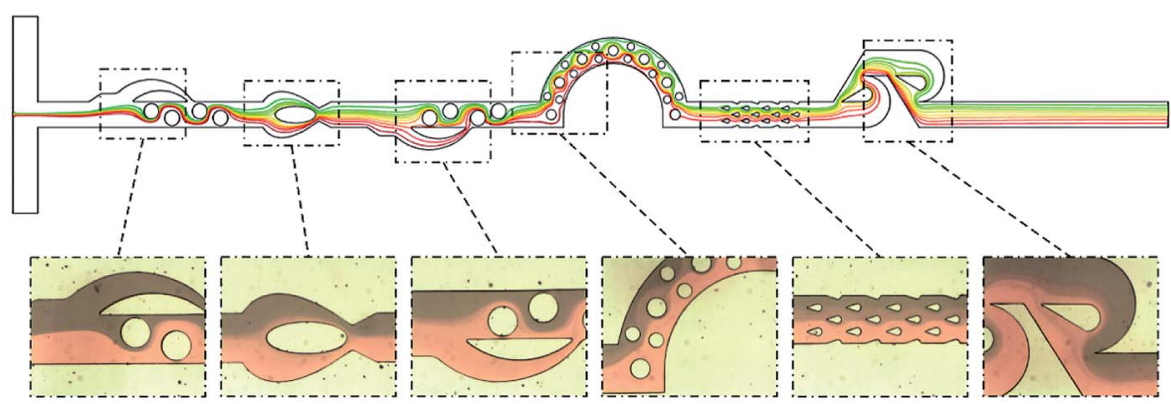

b)

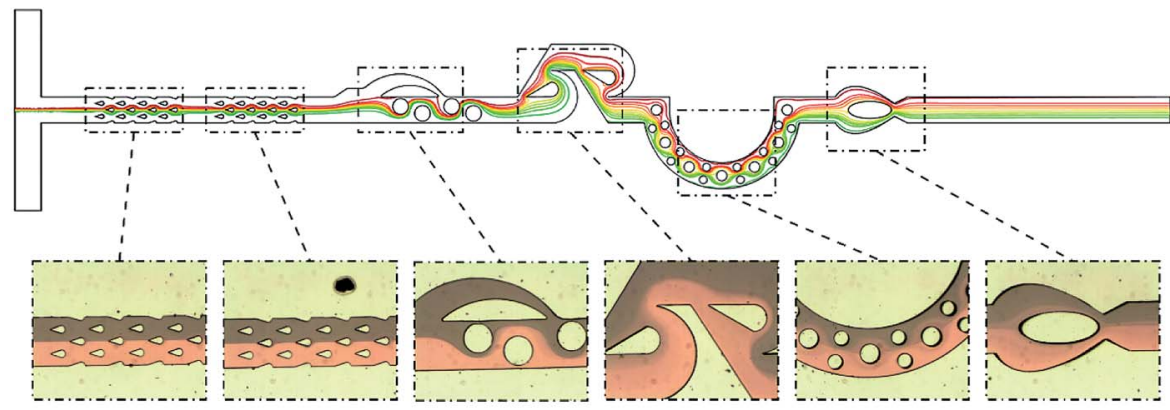

c)

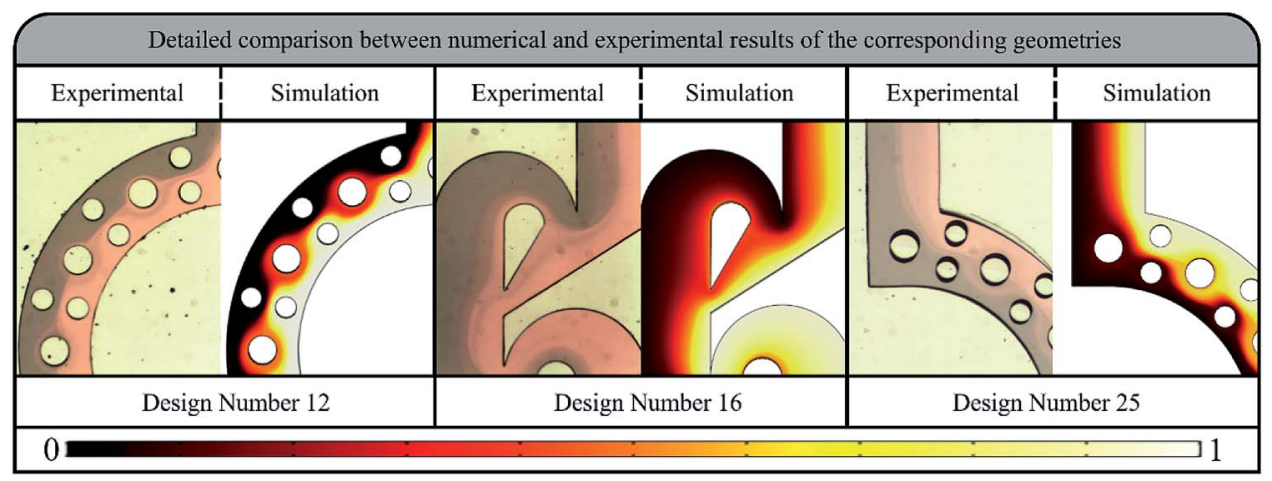

Fig. 5 Comparison of experimental and simulation results of (a) design number 12 (b) design number 16 and (c) design number 25 in terms of concentration distribution. Sectional views of each unit characterize the level of mixing present for both experimental and simulated tests.

accuracy of numerical results was proved through comparison, demonstrating that these findings will be of interest to the researchers working in this field (the video of these experimental results are provided in ESI $\dagger$ ).

\subsection{The "hybrid" micromixer with MURO}

An analysis was performed on the signal to noise $(\mathrm{S} / \mathrm{N})$ ratio diagram (Fig. 6) to find the best-nominated design from the proposed six factors and five levels. There are three classes of 


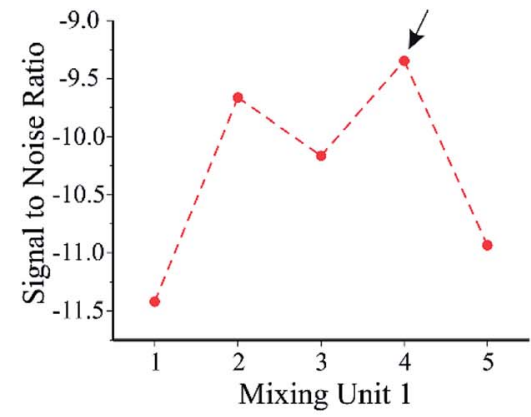

a)

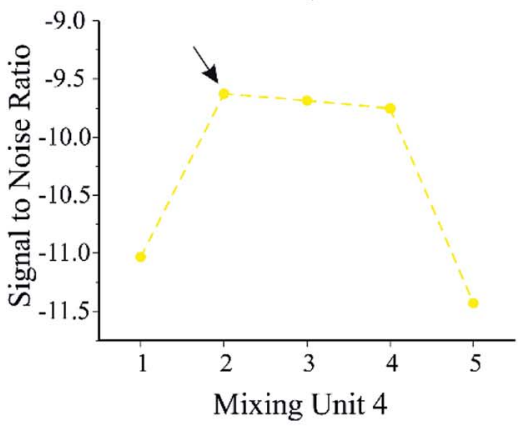

d)

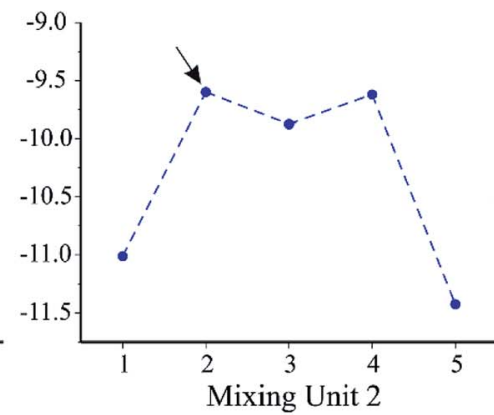

b)

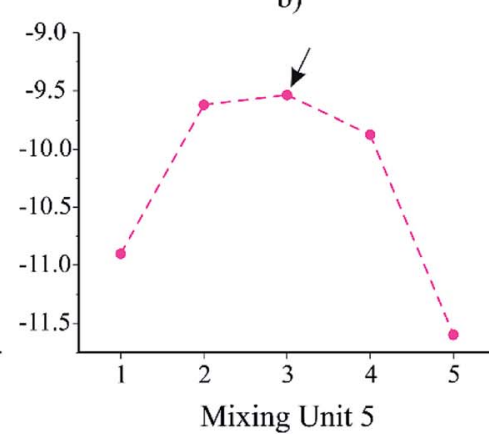

e)

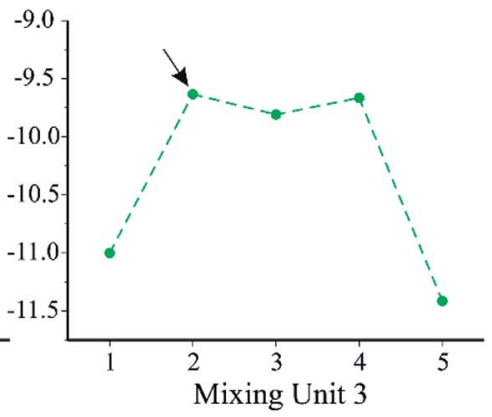

c)

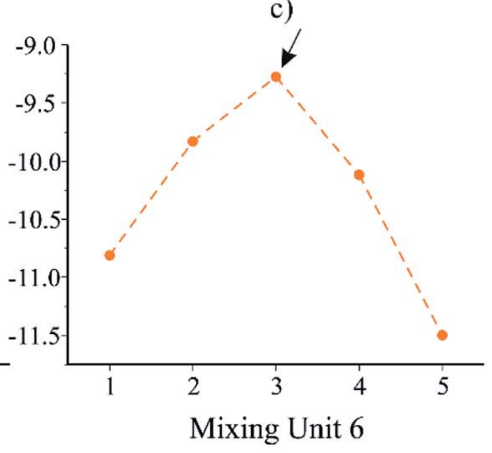

f)

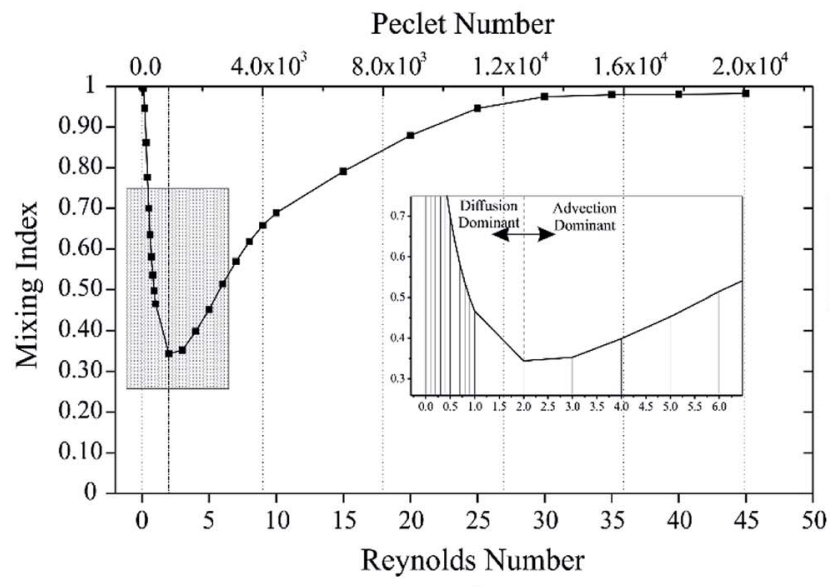

g)

h)

Fig. 6 (a-f) S/N ratio diagrams used to select the desirable mixing units. Points that are distinguished by black arrows indicate the highest $\mathrm{S} / \mathrm{N}$ ratio and the appropriate level for each slot (g) Mixing index of the hybrid micromixer in a wide range of Re and Pe is presented. The magnified section illustrates the change from diffusion dominated mixing to advection dominated mixing over Re of 0.001 to 6 (h) the optimized micromixer is portrayed with nominated MURO which are one nozzle and pillar mixing unit, three obstacle in a curved mixing units, and two Tesla mixing units.

quality in the analysis of $\mathrm{S} / \mathrm{N}$ ratio which categorically states that the-lower-the-better, the-nominal-the-better, and the-higherthe-better. The criterion for this study was set as the-higherthe-better, since the higher value of the mixing index increased the desirability of the "hybrid" micromixer. Eqn (13) shows the corresponding formula. ${ }^{34}$

$$
\mathrm{S} / \mathrm{N} \text { ratio }=-10 \times \log _{10}\left(\frac{1}{n} \sum_{i=1}^{n}\left(\frac{1}{Y^{2}}\right)\right)
$$

where, $n$ is the number of responses in the factor level combination and $Y$ is the responses for the given factor level combination. Fig. $6(\mathrm{a}-\mathrm{f})$ demonstrate $\mathrm{S} / \mathrm{N}$ ratio diagrams for $\mathrm{Re}=5$. The nominated mixing unit for each slot is the highest point in each factor in the $\mathrm{S} / \mathrm{N}$ ratio diagram which are specified by black arrows. As illustrated by Fig. 3(a-f), units 1 and 5 in each factor did not play a significant role on the output of the analysis. But, units 2,3 , and 4 in each factor had a chance to be nominated in the optimized micromixer. The "hybrid" micromixer has six separate units with this sorted arrangement:

Hybrid micromixer: mixing unit $3+($ mixing unit 2$) \times 3+($ mixing unit 3) $\times 2$

As can be concluded from Fig. 6(a-f), unit 1 and unit 6 are more sensitive to the change of mixing units in comparison to other units. The sensitivity of six mixing units can be ranked as below: 
unit $1 \approx$ unit $6>$ unit $2 \approx$ unit $3 \approx$ unit $5>$ unit 4

Therefore, there are more variations in units 1 and 6 compared with other units. The "hybrid" micromixer with MURO design as well as its mixing efficiency across a range of Re is clarified in Fig. 6 ( $\mathrm{g}$ and $\mathrm{h}$ ). Various mixing tactics exist in the "hybrid micromixer". The first unit in the "hybrid micromixer" is the nozzle and pillar mixing unit which contains an asymmetric sequence of obstacles (offering more effective mixing when compared to linearly arranged obstacles ${ }^{35}$ ) to enhance the mixing efficiency and a micronozzle to minimize the diffusion length. The second, third, and fourth mixing units are curved mixing units with in-built obstructions which involve embedded barriers in a curved microchannel to increase the mixing efficiency. In the curved channels, fluids experience a unique application of inertial and viscous forces for the generation of a transverse flow field (Dean flows). Dean flows enhance diffusive transport and increase mixing quickly over short lengths. The mixing index can also be improved with embedded barriers. Obstacles in microchannels can agitate the fluids which leads to the generation of lateral mass transport to further increase the mixing efficiency. The fifth and the sixth mixing units are Tesla units which make use of the "Coanda effect". (Please refer to ESI $\dagger$ for the detailed description of Coanda effect in Tesla unit) Due to these effects, the "hybrid micromixer" is able to increase the mixing efficiency considerably.

Based on eqn (12), locating the critical point in the mixing index diagram is essential to investigating the effects of Re on the mixing index. The simulations were performed with Re $0.001,0.01,0.1,0.2$, (with increment of 0.1), 1, 2, (with increment of 1), 9, 10, 15 to 45 (with increment of 5). As noted above, the mixing process is divided into two separate parts. These two parts are separated at the critical point: the point where the minimum mixing index takes place. The location of the critical point depends on the velocity profile and geometry design of the microchannel and signals the mixing mechanism has changed from diffusion to mass advection. Before the critical point, the mixing process mainly relies on the diffusion where the velocity inverse varies with the mixing index. For a value of Re less than 0.1 , the velocity is small enough for the two fluids to mix together completely. As the velocity increases, Pe increases, and according to eqn (12), which associates the mixing process with a specific length, the mixing procedure becomes harder. This trend continues until it reaches the critical point, where the mixing mechanism changes. As Fig. $6 \mathrm{~g}$ demonstrates, the critical point is at $\operatorname{Re}=2$. After the critical point, the mechanism of mixing changes to mass advection, where the velocity varies proportionally with the mixing index. After a specific value for Re, which tends to be 35 in this case, complete mixing takes place.

Fig. 7(a and b) represent a detailed diagram of the mixing processes for the two Re of 0.5 and 25, before and after the critical point, respectively. However, mere study of concentration is not enough to evaluate and entirely comprehend the mixing process. The concentration gradient is exploited to evaluate diffusion trends within the fluids, and the contours of concentration gradient distribution can denote the occurrence of diffusion; as reported by Fick's second law which is $\partial C / \partial t=$ $D \nabla^{2} C .^{36}$ Accordingly, if the distribution of the concentration gradient is adequate and homogenous on the transverse section, the diffusion path is shorter; therefore, it assists in the mixture of the fluids.

Fig. 7a depicts the mixing process based on the diffusion for Re 0.5 (please refer to the ESI $\dagger$ for the experimental results). The mixing scheme does not contain any chaotic advection, and it is solely based on molecular diffusion. As the concentration gradient distribution illustrates, this "hybrid" micromixer induces a more homogeneous gradient after each mixing unit in order to improve the quality of the mixing process. In the absence of mixing units, the mixing index of the micromixer will be significantly inadequate as the homogeneity of the concentration gradient will remain largely constant. As specified by Fig. $7(\mathrm{a}$ and $\mathrm{b})$, the $1^{\text {st }}, 2^{\text {nd }}$, and $3^{\text {rd }}$ units play a major role in the mixing process compared to the others, with the most effective unit being the $2^{\text {nd }}$. Fig. $7 \mathrm{~b}$ illustrates the mixing process of the mass advection dominant combined micromixer with Re 25. The cross-sections of C-C and D-D represent the phenomenon of "disarray" in the concentration and concentration gradient contours. From the cross-section of $\mathrm{C}-\mathrm{C}$ to $\mathrm{F}-\mathrm{F}$, the interface rotates 180 degrees clockwise, and at each crosssection, in a specific region, the interface of the two fluids swings. This leads to a heightened level of chaotic advection and a decrease in the diffusion path, enhancing the mixing index. Moreover, the "saddle-shaped" pattern demonstrated in the concentration gradient distribution leads to exponential growth in the contact surface, shortening the diffusion path. ${ }^{37}$ These mentioned phenomena significantly improve the mixing index, allowing it to reach a value of $94.6 \%$ by the end of unit 6 . Fig. $7 \mathrm{~b}$ clarifies that the second mixing unit has the most impact on the output compared with other units. (Please refer to ESI, $\dagger$ for video of experiments of this nominated device) To better understand the effect of each unit, Fig. 7(c and d) show the mixing index of the nominated design versus the length of micromixer.

Fig. 7 (c and d) are divided into two parts. The first is accountable for the diffusion dominant mixing process and the second is representative of the advection-based mixing process. The range of Re values in Fig. 7c correspond to mass diffusion, producing an increasingly negative mixing index. Whereas the range of Re values in Fig. $7 d$ correspond to the presence of mass advection resulting in an increasing mixing index. This is due to the increase in the velocity, which enhances the tendency of fluids to become entirely mixed. An extensive analysis on Fig. 7 (c and d) shows that unit 2 has the most effect on the mixing index, while the last unit has the least effect. Furthermore, by increasing the velocity, complete mixing is achieved before the fluids reach the end. In other words, the mixing length will be decreased. There is no doubt that beyond $\operatorname{Re} 50$, the role of the last unit is negligible and unit 2 plays a significant role in the mixing process. Further investigating unit 2, Fig. 8(a-h) represent the contour plot diagram of the stream-wise vorticity in the YZ-plane for Re 25. 


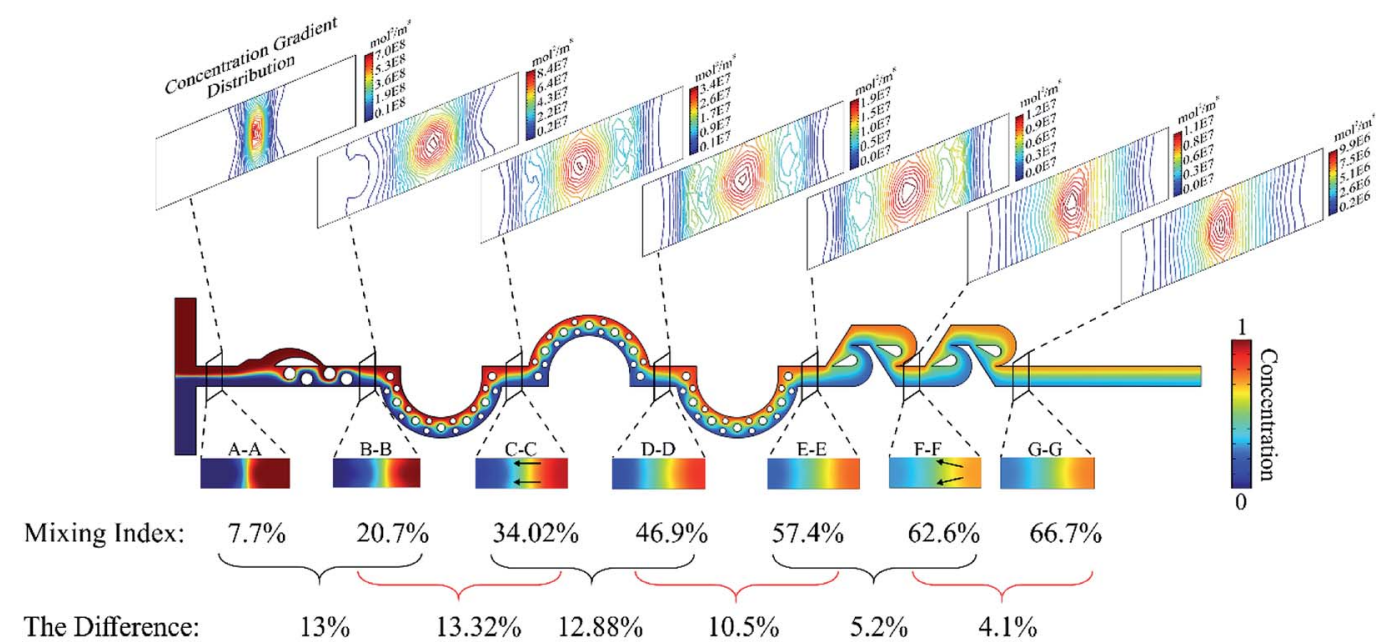

a)

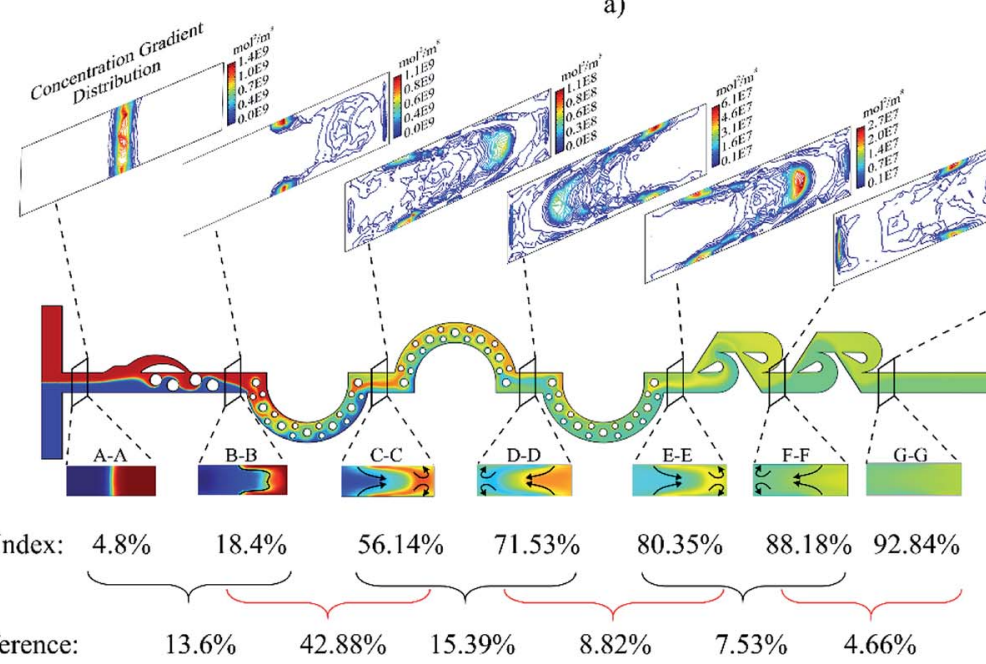

b)

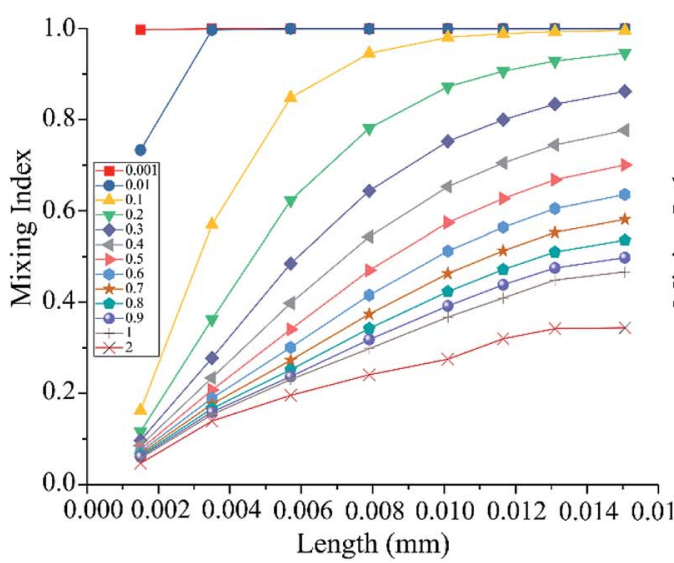

c)

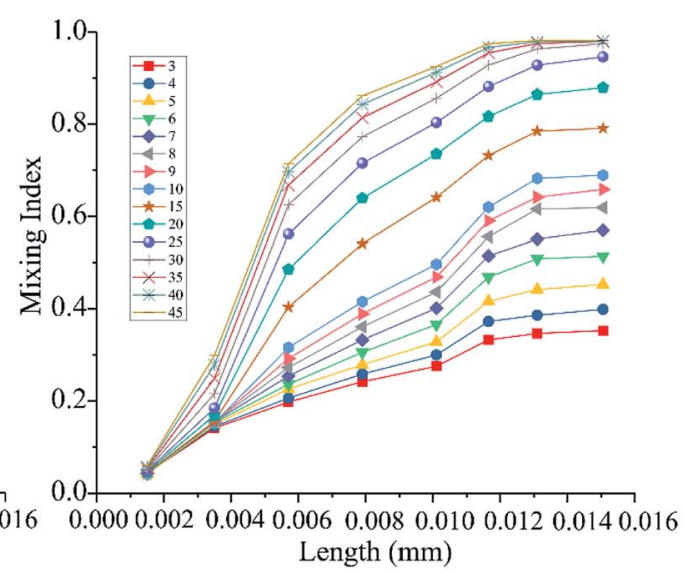

d)

Fig. 7 Distribution of concentration and concentration gradient at each cross-section as well the mixing index for (a) Re 0.5 and (b) Re 25 is depicted. For Re 25, the dislocation of the concentration gradient at each cross-section results in an increased mixing index up to $92 \%$ at the outlet. Black arrows indicate the presence of chaotic advection generated (c) mixing index versus the length of the "hybrid" micromixer for diffusion-based (d) advection-based mixing process is displayed. Legend shows Re.

For better illustration, a normalized length of unit $2\left(L_{\mathrm{N}}\right)$ was used. The vorticity in the direction of $X\left(\omega_{x}\right)$ can be calculated from eqn (14).

$$
\omega_{x}=\left(\frac{\partial v_{z}}{\partial y}-\frac{\partial v_{y}}{\partial z}\right)
$$




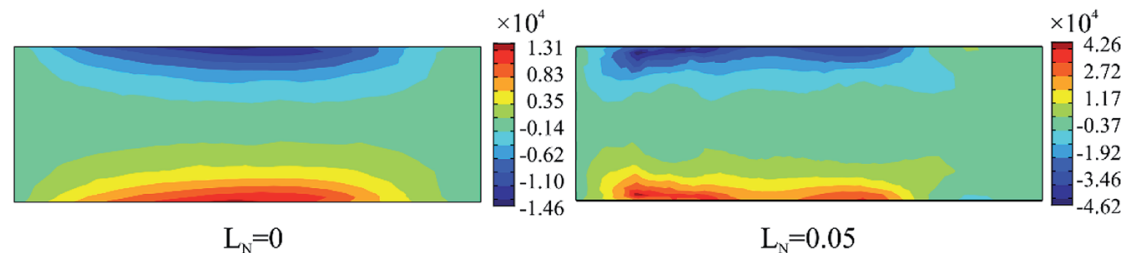

a)

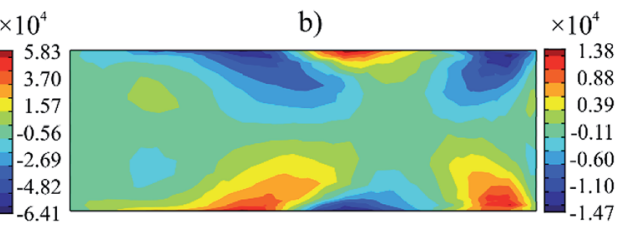

$\mathrm{L}_{\mathrm{N}}=0.22$

$\mathrm{L}_{\mathrm{N}}=0.45$

c)

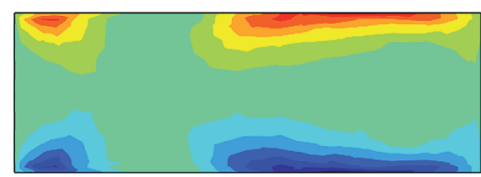

$\times 10^{4}$

d)

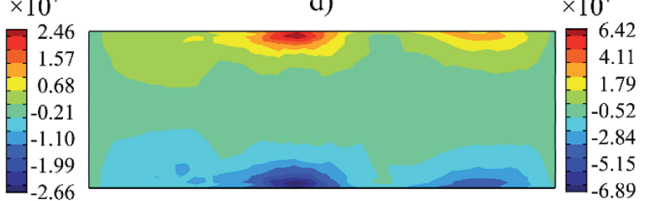

$\mathrm{L}_{\mathrm{N}}=0.64$

$\mathrm{L}_{\mathrm{N}}=0.78$

e)

f)

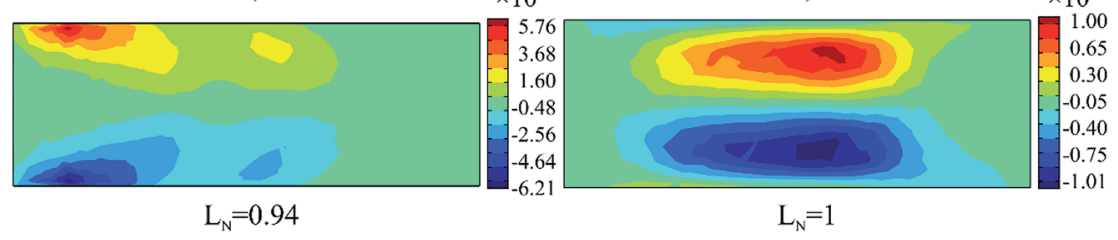

g)

h)

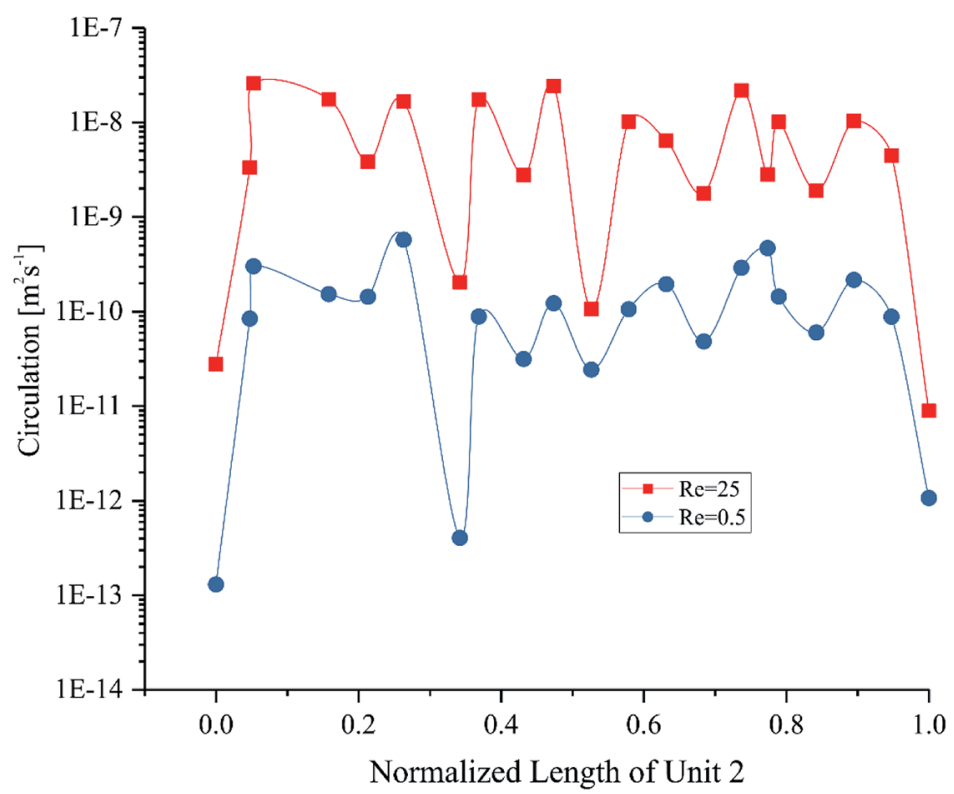

i)

Fig. $8(a-h)$ The contour diagram of the vorticity in $X$ direction of unit 2 with normalized length is presented. The peak vorticity relocates along the length of unit 2 (i) Values of circulation along the normalized length of unit 2 for both Re of 0.5 and 25 is shown.

where, $v_{z}$ and $v_{y}$ are velocity in the $Z$ and $Y$ directions, respectively. In Fig. $8(\mathrm{a}-\mathrm{h})$ the peak intensity regions are illustrated with two distinct colors at the starting point of unit 2. As the length increases, the peak intensity region appears near the walls and the level of $\omega_{x}$ elevates until it reaches a point which is near to the middle of the unit. Afterwards, the high vorticity regions change their position to the corresponding low vorticity regions, and there is a sudden drop in the peak intensity. Then, 
as the flows pass, the level of intensity becomes higher and the peak intensity region focuses on the walls. Just before the outlet, the peak intensity concentrates at the center of the channel and its peak decreases suddenly. The effect of changing the direction of the peak intensity and varying the level of the vorticity are the significant features of unit 2 that increase its mixing efficiency. ${ }^{38}$

The circulation of the sections in the $Y-Z$ plane as illustrated in Fig. 8a-h, for Re 0.5 and 25 are depicted in Fig. $8 \mathrm{i}$ as a semilogarithmic diagram. The circulation in the $Y-Z$ plane can be defined by eqn (15).

$$
\left|\Gamma_{x}\right|=\iint_{S}\left(\frac{\partial v_{z}}{\partial y}-\frac{\partial v_{y}}{\partial z}\right) \mathrm{d} y \mathrm{~d} z
$$

At the inlet, the circulation is low but increases rapidly as the length increases. Chaotic advection, which is induced by obstacles in the curved microchannel of unit 2, is related to the increased value of the circulation. Re is directly proportional to the intensity of the circulation. The fluctuation in the diagram can be attributed to the change in the direction of the peak vorticity which occurs near the middle of unit 2 . After the flow reaches the end of unit 2 , the value of circulation drops to its lowest point since no obstacles are present to disrupt the flow.

For further evaluation of the overall function of the "hybrid" micromixer Fig. 9 portrays the velocity diagram of each crosssection, before and after each mixing unit. (Please refer to the ESI $\dagger$ for evaluation of Tesla units in the "hybrid" micromixer). The velocity field in a rectangular channel for Poiseuille flow is described by eqn (16), while contour plots of the velocity field along symmetrical axes are depicted in Fig. 9a.

$$
v_{x}(y, z)=\frac{4 h^{2} \nabla p}{\pi^{3} \mu L} \sum_{n, \text { odd }}^{\infty} \frac{1}{n^{3}}\left[1-\frac{\cosh \left(n \pi \frac{y}{h}\right)}{\cosh \left(n \pi \frac{w}{2 h}\right)}\right] \sin \left(n \pi \frac{z}{h}\right)
$$

As expected, at cross-section of $\mathrm{A}-\mathrm{A}$, the velocity diagram resembles microscale flow in a rectangular channel. After the fluids pass the first mixing unit, the peak velocity changes and relocates to the outer wall of the microchannel which is around $0.5 \mathrm{~W}$. From here on, the peak velocity continually varies between the $\mathrm{C}-\mathrm{C}$ and $\mathrm{F}-\mathrm{F}$ cross-sections. The corresponding circumstances are mainly related to the sequential

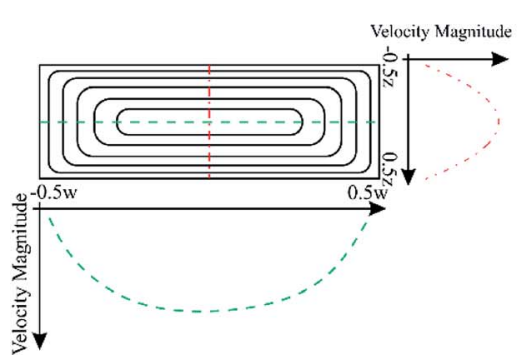

Detailed diagram
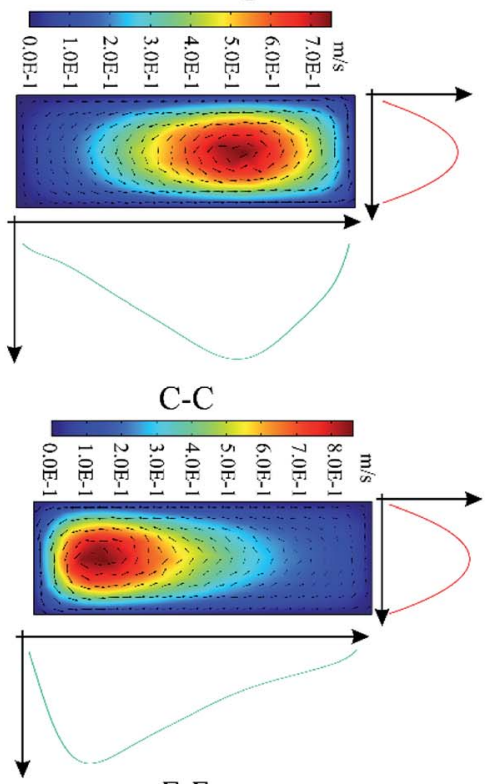

F-F

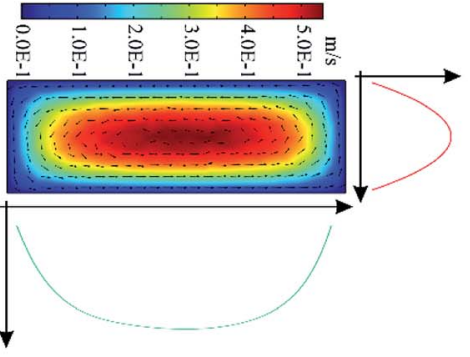

A-A

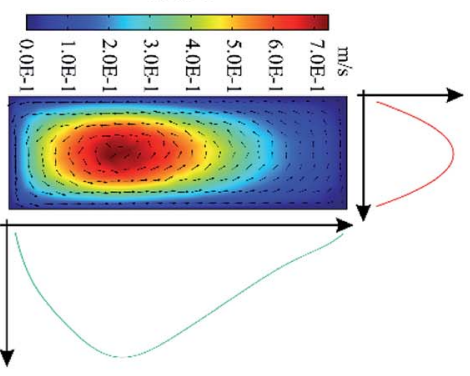

D-D

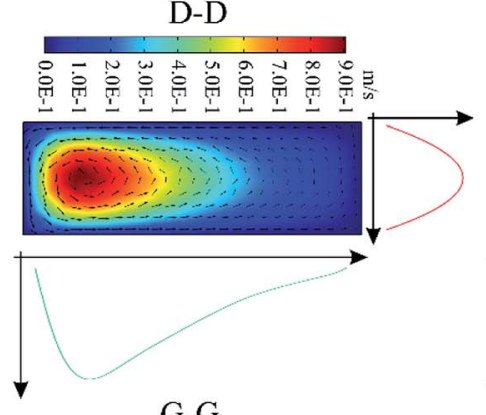

G-G

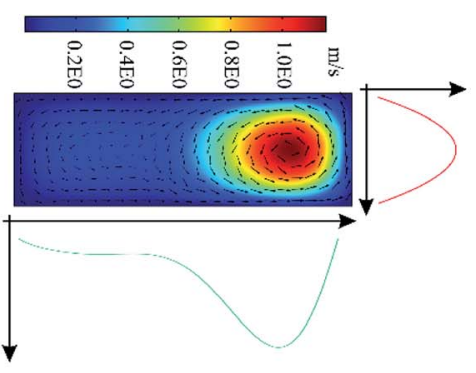

B-B

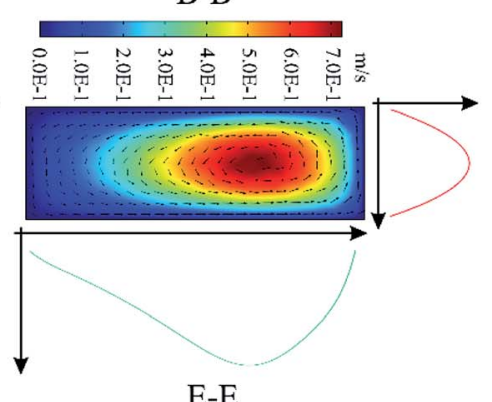

E-E

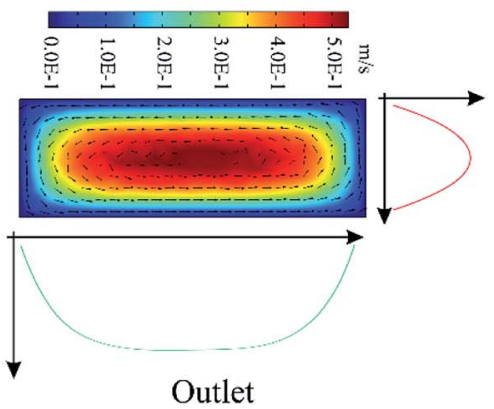

Fig. 9 Velocity diagram of different cross-sections of the "hybrid" micromixer with two velocity profiles at the symmetric axes. Along the height of the cross-section, the velocity distribution does not change. However, the velocity distribution along the width of the cross-section is influenced by the techniques employed by each MURO. 
Table 2 Different examples of micromixers are illustrated with their specific function and their comparison with the "hybrid" micromixer. The comparison was based on the mixing index reported in each study

\begin{tabular}{lll}
\hline Category & Examples & Features of the device \\
\hline Chemical synthesis & $\begin{array}{l}\text { Identification of } \\
\text { glucose-catalyst } \\
\text { reactions }\end{array}$ & $\begin{array}{l}\text { Mixing efficiency of } 81 \text { to } 92 \% \text { for Re } \\
\text { of } 0.08 \text { to } 16\end{array}$ \\
& $\begin{array}{l}\text { Active mixer for } \\
\text { loading of drug } \\
\left.\text { on } \mathrm{TiO}_{2} \text { (ref. } 39\right)\end{array}$ & $\begin{array}{l}\text { Mixing efficiency about } 90 \% \text { at Re of } \\
\text { 0.006. The device also had low } \\
\text { mixing index in high Re }\end{array}$
\end{tabular}

Synthesis of organic samples ${ }^{6}$

Chemical reactors

Polymerization

Sample concentration

Biological analysis

Wide ranges of applications
A micromixer with nozzle and pillar mixing units for DNA ligation $^{7}$ Polymerization
of vinyl ethers

Using unbalanced split and recombine mixing units for sample proteins ${ }^{41}$ Square-wave blood plasma mixing ${ }^{42}$ Formation of thrombosis $^{8}$

Drug screening with a zig-zag shaped micromixer $^{9}$ "Hybrid" micromixer preparation mixing units for
Comparison with the Corresponding Study

As the "hybrid" micromixer has an efficiency of $100 \%$, it can facilitate the process of detection in low and high Re (0.001-0.1 and 22-45 respectively)

The proposed micromixer has the mixing efficiency of $100 \%$ in Re of 0.001-0.1. Furthermore, it has a favorable function on high Re where the mixing efficiency in about $100 \%$

Using the "hybrid" micromixer can be effective in enhancing the mixing index, and by doing so, yield efficiency will be improved The "hybrid" micromixer proposes a mixing efficiency of $100 \%$ in high $\mathrm{Re}$, with significant function in low Re which can improve the efficiency of the device for the proposed application

The "hybrid" micromixer which employs different mixing tactics can have a mixing efficiency of $100 \%$ in high and low Re

investigated with different parameters where mixing index changes from 55 to $99 \%$ in different cases

Mixing index was reported as 35\%

in low Re while it reached the $85 \%$ in high Re

The minimum efficiency of the "hybrid" micromixer is more than this study. Therefore, it can easily be used for wide ranges of Re, and in low and high Re, the mixing efficiency is $100 \%$

The mixing index was reported as $76 \%$ for the optimized one

The "hybrid" micromixer can perform this experiment in low Re where the mixing index is $100 \%$

Using an active micromixer

Maximum mixing efficiency of $79.4 \%$ in the experimental test was reported

Since the active forces might be hazardous to biological samples, "hybrid" micromixer can readily be adapted to this aim

The proposed micromixer can produce results with a mixing index of $100 \%$ in low and high Re

Based on the comparison of mixing index of different devices, the "hybrid micromixer" offers complete mixing in Re 0.001-0.1, 40-45 and more than $90 \%$ mixing index in Re 22-40 which can be used for various bio-applications in either low or high ranges of $\mathrm{Re}$

upward/downward orientation of mixing units. This phenomenon induces further chaos and vortices at the transverse section and decreases the diffusional path, increasing the mixing index. As Fig. 9 reveals, after the crosssection of E-E, due to the fact that no mixing unit exists, the flow pattern adopted a rectangular flow pattern like that in AA. The direction of the vorticity field confirms the above situations. The main feature of the "hybrid" design is the combination of three different mixing units, each of which has unique features that when combined provide maximum mixing efficiency.

In order to evaluate the function of the "hybrid" micromixer in terms of its bio-applications, Table 2 compares the mixing efficiencies and Re for previously reported micromixers against the current "hybrid" micromixer for certain applications.

Table 3 details the properties of various named micromixers and provides a comparative analysis against the "hybrid" micromixer, in terms of Re range and mixing index. 
Table 3 Summary of certain micromixers with MURO and qualitative and quantitative comparison with the "hybrid" micromixer ${ }^{a}$

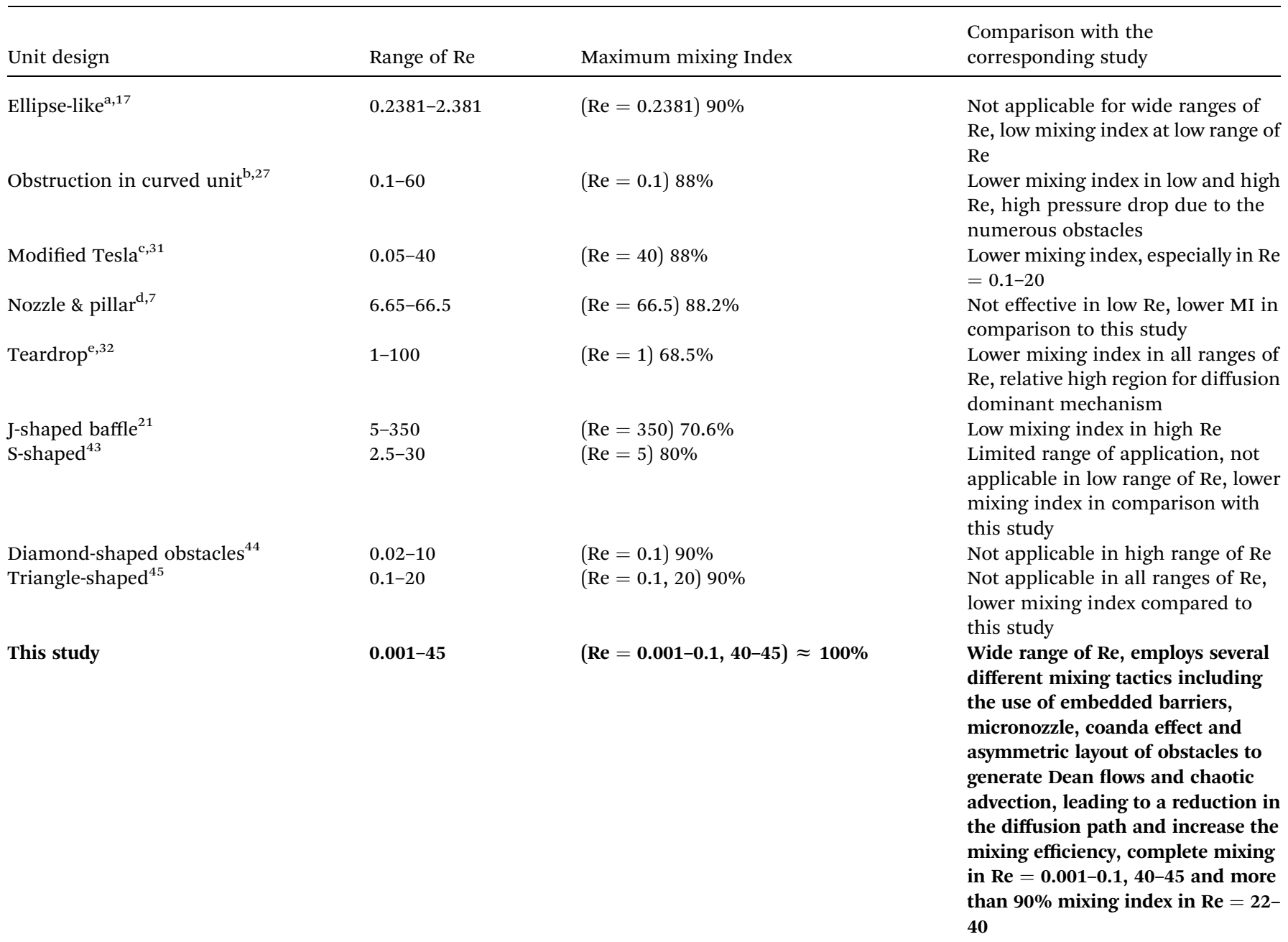

${ }^{a}$ a, b, c, d, e - these studies are account for mixing units $1,2,3,4$, and 5.

These comparisons reveal that the proposed "hybrid" micromixer can not only be employed in a wide variety of bioapplications at different Re, but also exerts different mixing methods due to the combination of different mixing units. Furthermore, it becomes clear that instead of using the complex 3D structures of micromixers, the proposed planar micromixer can be used for a range of 0.001-0.1 and 40-45 Re with a maximum mixing efficiency of $100 \%$. In addition, in other ranges of Re, the proposed micromixer indicated better or comparable results for application in comparison with other MURO. Altogether, the superior function of the "hybrid" micromixer composed of nominated MURO is apparent in both the detailed investigation regarding vortex, circulation, velocity profile, concentration and concentration distribution, and in comparison with other micromixers. As the micromixers are ubiquitous for all microfluidic devices, we are of the opinion that the corresponding geometry can render the experiments of researchers more accurate and convenient especially when considering integrated systems, such as labon-a-chip devices.

\section{Conclusion}

The primary aim of this study was to form a "hybrid" micromixer with MURO for wide ranges of applications. After the investigation on MURO, five units of elliptical, Tesla, nozzle and pillar, and teardrop mixing units, as well as a curved mixing unit with in-built obstructions were selected. Then, six slots were designated to put the mixing units in. As the combination of all these units leads to a total of 15625 experiments, Taguchi method as an appropriate DOE method was applied for the optimization process and reduced the total number of experiments to 25. As these units are not alike in terms of dimensions, the methods of dynamic similarity (an investigation about the dimensionless number) and geometric similarity (an investigation about mere dimensions of the units) were performed. The comparison illustrated that the numerical results could efficiently predict the experimental ones, and the flow behavior is the same in two cases. The optimized micromixer, termed the "hybrid micromixer" had six mixing units in which one was a nozzle and pillar mixing unit, three were obstacles in a curved 
unit, and two were modified Tesla units. The "hybrid" micromixer was tested for Re ranging between 0.001 and 45, and the results indicated that the mixing index first decreased as it approached the critical point, but later increased. This critical point divided this diagram into two separate parts. For the mentioned micromixer, Re 2 was related to the critical point, where the mixing mechanism changed from diffusion to advection process. The function of the "hybrid" micromixer in terms of mixing efficiency was considerable as the mixing index in the range of $0.001-0.1$ and $40-45 \mathrm{Re}$ is approximately $100 \%$ and in the range of $22-40$ is greater than $90 \%$. For further investigation of the micromixer, the precise function of each unit was investigated by the velocity profiles, concentration and concentration distribution diagrams, and mixing index, before and after each unit. The results demonstrated that the second unit has the highest impact on the mixing index, especially where advection is dominant in the mixing process. For better understanding, contour plots of vorticity and circulation along the length of the second unit have been displayed, and its function was evaluated. Since micromixers have various applications in microfluidic devices, a comparison of the "hybrid" micromixer with other micromixers in terms of mixing efficiency reveals that this micromixer can be applied for wide ranges of bio-applications. Since the suggested micromixer contains different mixing units which lead to a combination of mixing techniques, it would be an appropriate choice for future studies.

\section{Conflicts of interest}

There are no conflicts to declare.

\section{References}

1 A. Lashkaripour, R. Silva and D. Densmore, Desktop micromilled microfluidics, Microfluid. Nanofluid., 2018, 22(3), 31.

2 F. A. Gomez, Biological applications of microfluidics, John Wiley \& Sons, 2008.

3 N.-T. Nguyen and Z. Wu, Micromixers-a review, J. Micromech. Microeng., 2004, 15(2), R1.

4 C.-Y. Lee and L.-M. Fu, Recent advances and applications of micromixers, J. Micromech. Microeng., 2018, 259, 677-702.

5 D. Kim, et al., An easily integrative and efficient micromixer and its application to the spectroscopic detection of glucosecatalyst reactions, Analyst, 2005, 130(3), 293-298.

6 H. Kim, et al., Submillisecond organic synthesis: outpacing fries rearrangement through microfluidic rapid mixing, Science, 2016, 352(6286), 691-694.

7 Y.-J. Ko, et al., DNA ligation using a disposable microfluidic device combined with a micromixer and microchannel reactor, J. Micromech. Microeng., 2011, 157(2), 735-741.

$8 \mathrm{~T}$. Goerge, et al., Microfluidic reveals generation of plateletstrings on tumoractivated endothelium, Thromb. Haemostasis, 2007, 98(02), 283-286.
9 F. Nason, et al., Design of microfluidic devices for drug screening on in vitro cells for osteoporosis therapies, Microelectron. Eng., 2011, 88(8), 1801-1806.

$10 \mathrm{X}$. Chen and T. Li, A novel passive micromixer designed by applying an optimization algorithm to the zigzag microchannel, Chem. Eng. J., 2017, 313, 1406-1414.

11 S. Yu, T.-J. Jeon and S. M. Kim, Active micromixer using electrokinetic effects in the micro/nanochannel junction, Chem. Eng. J., 2012, 197, 289-294.

12 C.-Y. Lee, et al., Passive mixers in microfluidic systems: a review, Chem. Eng. J., 2016, 288, 146-160.

$13 \mathrm{~J}$. Zhang, et al., Fundamentals and applications of inertial microfluidics: a review, Lab Chip, 2016, 16(1), 10-34.

14 M. Mollajan, S. R. Bazaz and A. A. Mehrizi, A Thoroughgoing Design of a Rapid-cycle Microfluidic Droplet-based PCR Device to Amplify Rare DNA Strands, J. Appl. Fluid Mech., 2018, 11(1), 21-29.

15 M. Rafeie, et al., An easily fabricated three-dimensional threaded lemniscate-shaped micromixer for a wide range of flow rates, Biomicrofluidics, 2017, 11(1), 014108.

16 S.-J. Park, et al., Rapid three-dimensional passive rotation micromixer using the breakup process, J. Micromech. Microeng., 2003, 14(1), 6.

17 N. Tran-Minh, T. Dong and F. Karlsen, An efficient passive planar micromixer with ellipse-like micropillars for continuous mixing of human blood, Comput. Methods Programs Biomed., 2014, 117(1), 20-29.

18 C.-C. Hong, J.-W. Choi and C. H. Ahn, A novel in-plane passive microfluidic mixer with modified Tesla structures, Lab Chip, 2004, 4(2), 109-113.

$19 \mathrm{X}$. Chen and J. Shen, Numerical and experimental investigation on splitting-and-recombination micromixer with E-shape mixing units. Microsystem Technologies, 2016: pp. 1-7.

$20 \mathrm{X}$. Chen and J. Shen, Simulation and experimental analysis of a SAR micromixer with F-shape mixing units, Anal. Methods, 2017, 9(12), 1885-1890.

21 Y.-C. Lin, Y.-C. Chung and C.-Y. Wu, Mixing enhancement of the passive microfluidic mixer with J-shaped baffles in the tee channel, Biomed. Microdevices, 2007, 9(2), 215-221.

22 C.-K. Chung and T. Shih, Effect of geometry on fluid mixing of the rhombic micromixers, Microfluid. Nanofluid., 2008, 4(5), 419-425.

23 C.-K. Chung and T. Shih, A rhombic micromixer with asymmetrical flow for enhancing mixing, J. Micromech. Microeng., 2007, 17(12), 2495.

24 C. Chung, C. Chang and C. Lai, Simulation and fabrication of a branch-channel rhombic micromixer for low pressure drop and short mixing length, Microsyst. Technol., 2014, 20(10-11), 1981-1986.

25 S. Hossain and K.-Y. Kim, Mixing analysis of passive micromixer with unbalanced three-split rhombic subchannels, Micromachines, 2014, 5(4), 913-928.

26 A. Nejat, et al., Unsteady pulsating characteristics of the fluid flow through a sudden expansion microvalve, Microfluid. Nanofluid., 2014, 17(4), 623-637. 
27 A. Alam, A. Afzal and K.-Y. Kim, Mixing performance of a planar micromixer with circular obstructions in a curved microchannel, Chem. Eng. Res. Des., 2014, 92(3), 423-434.

28 L.-S. Jang, et al., Transport of particle-laden fluids through fixed-valve micropumps, Microelectromechanical Systems, ASME MEMS, 1999, vol. 1, p. 503-509.

29 D. C. Duffy, et al., Rapid prototyping of microfluidic switches in poly(dimethyl siloxane) and their actuation by electroosmotic flow, J. Micromech. Microeng., 1999, 9(3), 211.

30 A. A. S. Bhagat and I. Papautsky, Enhancing particle dispersion in a passive planar micromixer using rectangular obstacles, J. Micromech. Microeng., 2008, 18(8), 085005.

31 S. Hossain, et al., Analysis and optimization of a micromixer with a modified Tesla structure, Chem. Eng. J., 2010, 158(2), 305-314.

32 K. J. Cook, Y. Fan and I. Hassan, Experimental investigation of a scaled-up passive micromixer with uneven interdigital inlet and teardrop obstruction elements, Exp. Fluids, 2012, 52(5), 1261-1275.

33 S. B. Islami and M. Khezerloo, Enhancement of Mixing Performance of Non-Newtonian Fluids using Curving and Grooving of Microchannels, J. Appl. Fluid Mech., 2017, 10(1), 127-141.

34 N. Solehati, J. Bae and A. P. Sasmito, Optimization of WavyChannel Micromixer Geometry Using Taguchi Method, Micromachines, 2018, 9(2), 70.

$35 \mathrm{H}$. Wang, et al., Optimizing layout of obstacles for enhanced mixing in microchannels, Smart Mater. Struct., 2002, 11(5), 662.

36 X. Feng, Y. Ren and H. Jiang, An effective splitting-andrecombination micromixer with self-rotated contact surface for wide Reynolds number range applications, Biomicrofluidics, 2013, 7(5), 054121.

$37 \mathrm{H}$. Xia, et al., Chaotic micromixers using two-layer crossing channels to exhibit fast mixing at low Reynolds numbers, Lab Chip, 2005, 5(7), 748-755.

38 M. A. Ansari, Parametric study on mixing of two fluids in a three-dimensional serpentine microchannel, Chem. Eng. J., 2009, 146(3), 439-448.

39 N. Veldurthi, et al., Computational fluid dynamic analysis of poly (dimethyl siloxane) magnetic actuator based micromixer, J. Micromech. Microeng., 2015, 212, 419-424.

40 A. Nagaki, et al., Polymerization of vinyl ethers initiated by dendritic cations using flow microreactors, Tetrahedron, 2015, 71(35), 5973-5978.

$41 \mathrm{~K}$. Anwar, et al., Integrated micro/nano-fluidic system for mixing and preconcentration of dissolved proteins, Microchim. Acta, 2011, 173(3-4), 331-335.

42 J.-N. Kuo, H.-S. Liao and X.-M. Li, Design optimization of capillary-driven micromixer with square-wave microchannel for blood plasma mixing, Microsyst. Technol., 2017, 23(3), 721-730.

43 P. Li, J. Cogswell and M. Faghri, Design and test of a passive planar labyrinth micromixer for rapid fluid mixing, $J$. Micromech. Microeng., 2012, 174, 126-132.

44 A. A. S. Bhagat, E. T. Peterson and I. Papautsky, A passive planar micromixer with obstructions for mixing at low Reynolds numbers, J. Micromech. Microeng., 2007, 17(5), 1017.

$45 \mathrm{~K}$. Conlisk and G. M. O'Connor, Analysis of passive microfluidic mixers incorporating $2 \mathrm{D}$ and $3 \mathrm{D}$ baffle geometries fabricated using an excimer laser, Microfluid. Nanofluid., 2012, 12(6), 941-951. 